\title{
Influence of separation structure on the dynamics of shock/turbulent-boundary-layer interactions
}

Received: 26 December 2020 / Accepted: 13 August 2021 / Published online: 14 September 2021 (C) The Author(s) 2021

\begin{abstract}
Shock/turbulent-boundary-layer interactions (STBLIs) are ubiquitous in high-speed flight and propulsion applications. Experimental and computational investigations of swept, three-dimensional (3-D) interactions, which exhibit quasi-conical mean-flow symmetry in the limit of infinite span, have demonstrated key differences in unsteadiness from their analogous, two-dimensional (2-D), spanwise-homogeneous counterparts. For swept interactions, represented by the swept-fin-on-plate and swept-compression-ramp-on-plate configurations, differences associated with the separated shear layers may be traced to the intermixing of 2-D (spanwise independent) and 3-D (spanwise dependent) scaling laws for the separated mean flow. This results in a broader spectrum of unsteadiness that includes relatively lower frequencies associated with the separated shear layers in 3-D interactions. However, lower frequency ranges associated with the global "breathing" of strongly separated 2-D interactions are significantly less prominent in these simple, swept 3-D interactions. A logical extension of 3-D interaction complexity is the compound interaction formed by the merging of two simple interactions. The first objective of this work is therefore to analyze the more complex picture of the dynamics of such interactions, by considering as an exemplar, wall-resolved simulations of the double-fin-onplate configuration. We show that in the region of interaction merging, new flow scales, changes in separation topology, and the emergence of lower-frequency phenomena are observed, whereas the dynamics of the interaction near the fin leading edges are similar to those of the simple, swept interactions. The second objective is to evolve a unified understanding of the dynamics of STBLIs associated with complex configurations relevant to actual propulsion systems, which involve the coupling between multiple shock systems and multiple flow separation and attachment events. For this, we revisit the salient aspects of scaling phenomena in a manner that aids in assimilating the double-fin flow with simpler swept interactions. The emphasis is on the influence of the underlying structure of the separated flow on the dynamics. The distinct features of the compound interactions manifest in a centerline symmetry pattern that replaces the quasi-conical symmetry of simple interactions. The primary separation displays topological closure to reveal new length scales, associated unsteadiness bands, and secondary flow separation.
\end{abstract}

Keywords Swept shock/boundary layer interactions · Large-Eddy Simulations

Communicated by Sergio Pirozzoli.

M. C. Adler

Center for Turbulence Research, Stanford University, Stanford, CA 94305, USA

E-mail: mcadler@stanford.edu

D. V. Gaitonde $(\varangle)$

Mechanical and Aerospace Engineering, Ohio State University, Columbus, OH 43210, USA

E-mail: gaitonde.3@osu.edu 


\section{Introduction}

Shock/turbulent-boundary-layer interactions (STBLIs) are an unavoidable phenomenon for high-speed air vehicles. They occur on external surfaces as well as within the propulsion system and lead to a variety of design and performance constraints. Strong interactions result in flow separation that introduces new mechanisms of flow unsteadiness, including new frequencies observed two or more orders of magnitude below the characteristic frequencies of the associated turbulent boundary layers [22,24,25]. These new mechanisms of unsteadiness can include coupling between multiple shock systems, coupling between multiple flow separation and attachment events, coupling with the structural dynamics of the vehicle, and coupling with the combustion processes, each presenting additional challenges. Recent developments and remaining fundamental challenges in this area are broadly discussed by Clemens and Narayanaswamy [20] and Gaitonde [28].

Over the last decade or so, significant advancements have been achieved in the understanding of the dynamics of spanwise-homogeneous (nominally two-dimensional: 2-D) STBLIs. Commonly interrogated configurations include the quasi-infinite impinging-shock (IS: frame a) and compression-ramp (CR: frame b) interactions, as illustrated in Fig. 1. A variety of experimental (e.g., Souverein et al. [75]) and computational (e.g., Morgan et al. [52]) efforts have explored these interactions, and the complicated dynamics have been successfully described from several complementary perspectives. Some descriptions place emphasis on the upstream forcing of the interaction $[16,33,63]$, others the timescales associated with the separation dynamics [57,58], downstream Görtler-like forcing [65], the filtering behavior of the interaction [79], the oscillator behavior [60], or the absolute instability of the flow [55,59,78].

We recently discussed a unified mechanism [3] that may be broadly classified as a nonlinear oscillator, which exhibits linear restoring tendencies at extreme shock displacements consistent with the linear oscillator model [60], while exhibiting asymmetry with respect to the mean shock position consistent with the absolute instability description $[55,59,78]$. The nonlinearity (which is necessary for oscillator asymmetry) is a consequence of the external forcing, which likely results from a combination of upstream $[16,33,63]$ and downstream $[57,65]$ mechanisms.

\section{2-D interactions (spanwise homogeneous symmetry)}

a) Impinging shock

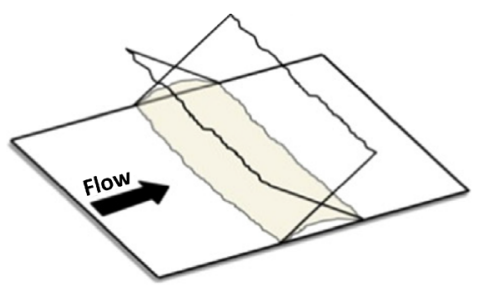

b) Compression ramp

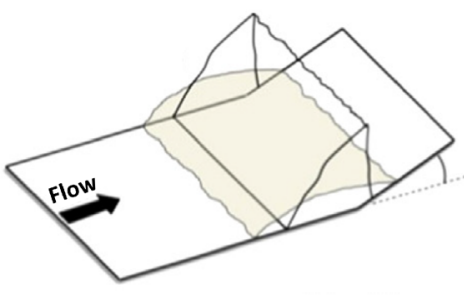

\section{Compound 3-D interactions (complex symmetry)}

e) Double fin

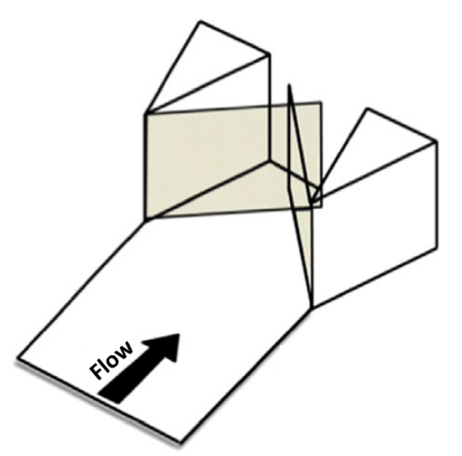

c) Swept compression ramp

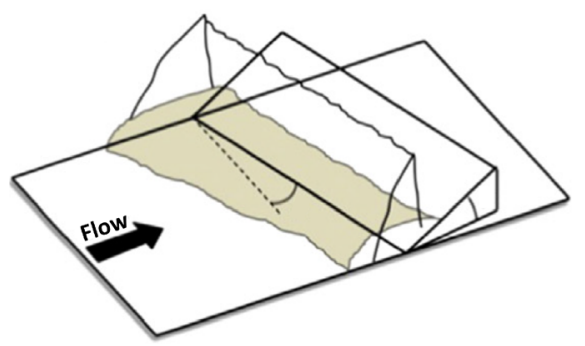

d) Sharp fin

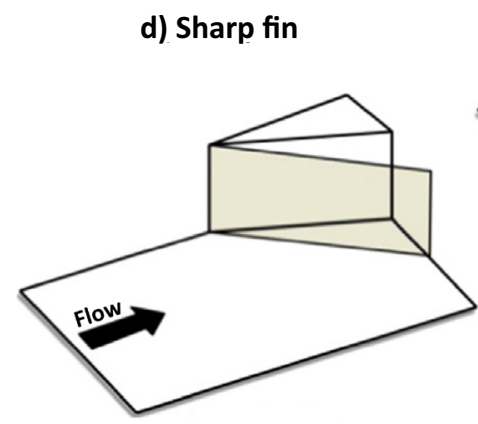

(quasi-conical symmetry)

Fig. 1 Principal STBLI configurations examined. a Impinging shock b and compression ramp, c swept compression ramp, d sharp fin, e symmetric double fin 
Ongoing work relevant to pseudo-2-D STBLIs includes recent exploration of confinement effects due to sidewalls on otherwise spanwise-homogeneous interactions. These confinement effects lead to significant changes in the separation structure $[62,66,85]$, which could consequently alter the dynamics. Therefore, a complete dynamical description of highly confined pseudo-2-D interactions should also be pursued.

Several three-dimensional (3-D) STBLIs (in which the mean flow is not spanwise homogeneous) have also been interrogated over the years [86]; however, the development of the dynamical description of these interactions has received significantly less attention than for their 2-D counterparts. A dynamical understanding of these simple and compound 3-D STBLIs is relevant to many aerospace applications, and affect crucial phenomena both internally (e.g., total pressure recovery in sidewall-compression inlets [40,54]) and externally (e.g., shock-induced flow separation for delta-wing vehicles [23,51]).

Simple, swept (3-D) interactions (in which the mean flow is quasi-conical in the limit of infinite span) have served as canonical configurations for both experimental [69-71] and computational (largely ReynoldsAveraged Navier-Stokes: RANS-based) investigations [46,56]. These include the swept-compression-ramp (SCR: frame c) and sharp-fin (SF: frame d) interactions, as illustrated in Fig. 1, for which mean-flow models were proposed by Knight et al. [44] (for the SF) and Knight et al. [45] (for the SCR). The quasi-conical meanflow symmetry of these interactions is realized through the approximate collapse of profiles plotted in spherical coordinates centered on a virtual conical origin (VCO) [72]. Visually, these interactions exhibit separation, attachment, and shock-triple-point lines that outline a conical structure, with each line emanating from the cone apex, which is located at the VCO. 3D effects of course can manifest in multiple forms that delineate other crucial features. For example, the swept-impinging-shock (SIS) is the subject of parallel experimental [21,77] and computational [39] efforts. Likewise, Zuo et al. [87] describe a conical SBLI containing a rich variety of unique 3-D effects. To limit the scope of the paper, these not considered here.

The merging, or compounding, of simple, swept interactions becomes important in finite-span configurations. A canonical example of such a compound swept interaction is the centerline-symmetric double-fin interaction (DF: frame e), illustrated in Fig. 1. A mean-flow model was proposed by Gaitonde et al. [30] and asymmetric variants displaying additional complexity have been discussed by Gaitonde et al. [29] and Thivet et al. [76]. The introduction of new scales may be expected to have corresponding implications on the characteristics of the dynamics, but have not been examined in detail in the archival literature (cf. Adler and Gaitonde [6], Adler [1]). The first objective of the present work is to fill this gap from analysis of high-fidelity simulations.

The compound, double-fin interaction presents several complications associated with interaction merging: most significantly, the additional unsteadiness and associated extrema in wall momentum and heat transfer associated with the secondary separation and attachment events. Though not exhibited by the STBLIs of our present databases, simple swept interactions are known to exhibit secondary separation under certain high-Mach-number conditions [56], whereas the compound double-fin interactions (including those of our databases) exhibit this phenomenon over a much larger parameter space.

While conceptually accurate mean-flow models of these simple and compound 3-D interactions were well described by the experimental and RANS-based synthesis of the 1980s and 1990s, limitations of the era prevented the acquisition of a detailed perspective. In computations, difficulties in RANS-based turbulence modelling are evident in efforts to predict quantitatively correct skin friction, heat transfer, and separation size, due to the highly compressible and nonequilibrium character of the turbulence. The dynamics of these interactions were also largely inaccessible at the time, with several notable experimental exceptions (e.g., Schmisseur and Dolling [67], Erengil and Dolling [26], Schmisseur and Dolling [68]).

Since that time, understanding of the dynamics of 2-D STBLIs has progressed significantly, and are often described with respect to different mechanisms acting across several frequency bands (see, e.g., Agostini et al. [9,10], Aubard et al. [13], Adler and Gaitonde [3]). The lowest prominent frequency band has been demonstrated to scale well across a variety of flow conditions, with Strouhal number $\left(S t_{L}=f L / U_{\infty} \sim 0.03\right)$ based on the peak low frequency $(f)$, the freestream velocity $\left(U_{\infty}\right)$, and the mean separation length $(L)$ [24]. The underlying mechanisms for this band of unsteadiness are multiply dependent and complex as mentioned previously. The highest frequency band naturally scales with $S t_{\delta} \simeq 1$, reflecting the underlying integral scales $(\delta)$ of the incoming boundary layer turbulence, the convection of which largely drive this frequency band. A significant middle frequency band is also observed $\left(S t_{\delta} \sim 0.1\right)$, typically one decade below the peak of the band describing fluctuations of the undisturbed boundary layer [10]. The mechanism underlying this frequency band relates to mean flow gradients in the post-separation shear layers, and the development of convective, vortical, coherent fluctuations in these shear layers, which have been described by several experimental $[74,75]$ and computational $[3,7,10,13,55]$ investigations. Therefore, this middle frequency band actually scales with 
local properties of the separated shear layer (see, e.g., Helm et al. [41], Adler and Gaitonde [7]), with $S t_{\delta} \sim 0.1$ providing a crude estimate based on global interaction properties.

Extending these concepts from 2-D to 3-D interactions presents significant challenges. Concerning the lowest frequency band of unsteadiness, while a constant global (span independent) separation length scale $(L)$ exists for spanwise-homogeneous interactions, the same is not true for simple swept interactions, due to their quasi-conical symmetry [5]; the separation length becomes a local (not global) property of the flow. Therefore, the mechanism underlying the low-frequency band of unsteadiness in 2-D interactions, scaling with $S t_{L}$, must change, and it appears to be significantly muted in simple swept interactions [7]. Concerning the middle frequency band of unsteadiness, unlike spanwise-homogeneous interactions, which exhibit 2-D freeinteraction scaling [18,38], simple swept interactions exhibit a mixture of 2-D and 3-D [72] free-interaction scaling laws for the separated shear layer [5], which result in a mixture of 2-D and 3-D scaling laws for the resulting middle frequency band of unsteadiness [7]. The high-frequency band largely retains the $S t_{\delta} \sim 1$ scaling of the incoming turbulent boundary layer; however, this band is also slightly altered locally due to extreme compression or expansion and separation or attachment events.

As demonstrated in achieving the first objective of the present work, the compound double-fin interaction requires an even more complex description of all frequency bands due to the secondary separation and attachment events. Its dynamics offer an opportunity to generalize the spectral evolution from 2-D and simple 3-D to compound 3-D dynamics, and to delineate the corresponding changes in relationships between 2-D and 3-D scales; this forms the second objective of the paper. We thus conjoin a new dynamical description of the compound double-fin interaction to the increasingly complex sequence of spanwise-homogeneous 2-D and simple 3-D interactions, as shown in Fig. 1.

Some previous results from these campaigns have been described in a variety of forms, including experimental measurements of the swept-compression-ramp (SCR) [80-82] and sharp-fin (SF) $[11,12,15,42,49,50]$ interactions, along with high-fidelity large-eddy simulations [2,4-7] of both configurations. The double-fin flowfield fosters a better understanding of the separation structure-unsteadiness connection, since it combines elements of swept 3-D interactions with those of 2-D interactions - the symmetry plane, for example, shows the influence of both - and thus aids in the establishment of a more comprehensive perspective.

The simulation databases, including the flow parameters (2.1) and details of the numerical method (2.2), are summarized in Sect. 2. For completeness, properties of the simple, swept, 3-D interactions are then refreshed and recast for the present goals in Sect. 3, including aspects of mean-flow scaling laws (3.1) and their effect on interaction dynamics (3.2). Properties of the compound double-fin interaction are then addressed in Sect. 4, including details of the mean-flow structure (4.1) and an examination of the band-isolated dynamics (4.2). Section 5 describes the influence of the separated-flow structure on 2-D, simple 3-D, and compound 3-D interactions, with emphasis placed on how differences in separation structure correlate with those observed in dynamical behavior among each of the classes of interactions. Concluding remarks are made in Sect. 6 .

\section{Details of STBLI databases}

Although descriptions of the databases assimilated in this work are presented in different publications, we collect pertinent aspects together in this section for easy reference.

\subsection{STBLI configurations}

The flow and geometric parameters of each configuration referred to in this work are specified in Tables 1 and 2, respectively. These parameters are chosen to facilitate comparisons, documented in previous work, with concurrent or archival experiments, depending on availability. Specifically, impinging shock simulations [3] were performed in coordination with experiments by Webb et al. [84], swept-compression-ramp interactions [2,7] with experiments by Vanstone et al. [81,82], Vanstone and Clemens [80], and sharp-fin interactions [4,7] with experiments by Arora et al. [11], Baldwin et al. [15], Mears et al. [49], Arora et al. [12], Mears et al. [50], Jones et al. [42]. Since recent double-fin interactions are not available, these are performed based on conditions of archival experiments by Garrison et al. [36], Garrison and Settles [35] and archival RANS calculations by Gaitonde and Shang [27]. Because the flow parameters chosen for these simulations are predicated on existing or concurrent experiments performed in a diverse set of facilities, they span a range of Mach and Reynolds numbers. This affects the dimensional spatio-temporal scales observed in each interaction. However, as discussed in Sect. 1, the evidence for both 2-D [25] as well as simple, swept, 3-D interactions [7] suggests 
Table 1 Experimental and computational flow conditions for STBLI databases

\begin{tabular}{lllllllll}
\hline Property & Exp. IS & Sim. IS & Exp. SCR & Sim. SCR \& CR & Exp. SF & Sim. SF & Exp. DF & Sim. DF \\
\hline$M_{\infty}$ & 2.3 & 2.3 & 2.0 & 2.0 & 2.0 & 2.0 & 4.0 & 4.0 \\
$U_{\infty}\left(\frac{m}{s}\right)$ & 556 & 556 & 510 & 510 & 518 & 518 & 672 & 672 \\
$P_{\infty}(P a)$ & 23511 & 2351 & 33360 & 1560 & 44940 & 4560 & 9880 & 2582 \\
$T_{0}(K)$ & 296 & 296 & $292 \pm 5$ & 292 & $300 \pm 5$ & 300 & 295 & 295 \\
$\delta(m)$ & $5.3 \times 10^{-3}$ & $5.3 \times 10^{-3}$ & $12.5 \times 10^{-3}$ & $12.5 \times 10^{-3}$ & $3.4 \times 10^{-3}$ & $3.4 \times 10^{-3}$ & $2.87 \times 10^{-3}$ & $2.87 \times 10^{-3}$ \\
$\Theta(m)$ & $0.77 \times 10^{-3}$ & $0.77 \times 10^{-3}$ & $0.9 \times 10^{-3}$ & $0.96 \times 10^{-3}$ & N/A & $0.26 \times 10^{-3}$ & $0.128 \times 10^{-3}$ & $0.128 \times 10^{-3}$ \\
$\operatorname{Re}_{\delta}$ & 175200 & 17520 & 475000 & 16210 & 159800 & 16210 & 229600 & 60000 \\
$\operatorname{Re}_{\Theta}$ & 25390 & 2539 & 34200 & 1250 & N/A & 1250 & 12488 & 2676 \\
\hline
\end{tabular}

Table 2 Geometries of interest: angles of sweep/attack, compression, and inviscid flow deflection, with corresponding displacement of simulation virtual and inceptive origins from geometric origin

\begin{tabular}{lcccll}
\hline Geometry & Sweep/attack & Compression & Inviscid flow deflection & Virtual origin $(x, z) / \delta_{0}$ & Inceptive origin $(x, z) / \delta_{0}$ \\
\hline IS9 & $0.0^{\circ}$ & $9.0^{\circ}$ & $9.0^{\circ}$ & $(0,-\infty)$ & $(0,-\infty)$ \\
IS11 & $0.0^{\circ}$ & $11.0^{\circ}$ & $11.0^{\circ}$ & $(0,-\infty)$ & $(0,-\infty)$ \\
CR225 & $0.0^{\circ}$ & $22.5^{\circ}$ & $22.5^{\circ}$ & $(0,-\infty)$ & $(0,-\infty)$ \\
SCR225 & $30.0^{\circ}$ & $22.5^{\circ}$ & $21.9^{\circ}$ & $(-3.4,-5.8)$ & $(-3.9,-6.7)$ \\
SCR300 & $30.0^{\circ}$ & $30.0^{\circ}$ & $28.7^{\circ}$ & $(-2.2,-3.7)$ & $(-4.1,-7.8)$ \\
SCR375 & $30.0^{\circ}$ & $37.5^{\circ}$ & $35.1^{\circ}$ & $(-1.4,-2.4)$ & $(-5.8,-11.3)$ \\
SF15 & $15.0^{\circ}$ & $90.0^{\circ}$ & $15.0^{\circ}$ & $(-1.5,-1.5)$ & $(-5.0,-5.1)$ \\
SF22 & $22.0^{\circ}$ & $90.0^{\circ}$ & $22.0^{\circ}$ & $(-0.9,-1.5)$ & $(-3.9,-6.3)$ \\
DF15 & $15.0^{\circ}$ & $90.0^{\circ}$ & $15.0^{\circ}$ & $(-4.1,-2.6)$ & $(-6.3,-3.0)$ \\
\hline
\end{tabular}

Experimental comparison available for cases IS9, SCR225, SF15, and DF15. Inviscid shock detachment occurs at $\approx 23.0^{\circ}$ flow deflection for Mach 2. The compression angle for the impinging shock interaction describes the far field shock generator

that the unsteadiness mechanisms have relatively similar behavior across a range of Mach and Reynolds numbers. Particularly, for 3-D interactions, a larger parameter space should be investigated moving forward to better quantify the degree of similarity with respect to these properties [14]. Employing proper length and velocity scales for normalization facilitates the comparison of spatio-temporal scales of different mechanisms associated with each interaction type across a range of flow parameters.

The flow properties, Table 1, indicate simulation and respective experimental conditions for the impingingshock (IS), swept-compression-ramp (SCR), sharp-fin (SF), and double-fin (DF) interactions. The simulation Reynolds number based on freestream conditions and boundary layer 99\% thickness $\left(R e=\rho_{\infty} U_{\infty} \delta / \mu_{\infty}\right)$ is reduced to facilitate wall-resolved simulations, while maintaining the freestream Mach number $\left(M_{\infty}\right)$ and velocity $\left(U_{\infty}\right)$, the stagnation temperature $\left(T_{0}\right)$, and the boundary layer momentum $(\Theta)$ and outer-scale $\left(u(\delta)=0.99 U_{\infty}\right)$ thicknesses, and reducing the freestream pressure $\left(P_{\infty}\right)$. Validation with experiment [3,5] has demonstrated that this approach reproduces many mean-flow properties relatively independent of Reynolds number, including the shock structure, the separated shear layer scaling, and the locations of flow separation and attachment, as well as some dynamic features of the flow that are not significantly affected by Reynolds number, provided the incoming boundary layer thickness is properly matched (see also Morgan et al. [52]). Therefore, we anticipate the major conclusions relating separation structure to flow dynamics to be relatively independent of Reynolds number; though this postulate requires further investigation with large-Reynoldsnumber simulations. Wall-modeled LES can be an effective tool for assessing both the mean and dynamic properties of high-Reynolds-number STBLIs [17]; however, work is still required to examine simulations of swept STBLIs in the high-Reynolds-number regime.

The parameter space of the interaction geometry considered is described in Table 2. Two IS, one CR, three SCR, two SF, and one DF constitute the STBLI databases. The details of several relevant angles and the locations of the virtual (VCO) and inceptive (ICO) conical origins with respect to the geometric origin (the fin-tip/plate junction or the upstream-ramp-corner/side-boundary) are included. The VCO is the origin of conical symmetry, whereas the ICO describes the effects of the incoming boundary layer thickness on deviation from true conical symmetry; the two become coincident with the geometric origin in the limit of radius $(r)$ much larger than boundary layer thickness $(\delta)$. The dependence of the VCO and ICO position on interaction strength and mechanisms of interaction inception has recently been documented [5]. 
Table 3 Computational grid properties for 3-D interactions

\begin{tabular}{llll}
\hline Property & SCR & SF & DF \\
\hline$L_{x} \times L_{y} \times L_{z}$ in $\delta_{0}$ & $32 \times 25 \times 17$ & $36 \times 20 \times 24$ & $75.1 \times 9.7 \times(12.1-33.6)$ \\
$N_{x} \times N_{y} \times N_{z}$ & $633 \times 251 \times 590$ & $1010 \times 249 \times 675$ & $1457 \times 263 \times 681$ \\
$N_{\text {total }}$ & $8.93 \times 10^{7}$ & $1.70 \times 10^{8}$ & $2.61 \times 10^{8}$ \\
$\Delta x^{+} \times\left(\Delta y_{\text {min }}^{+}-\Delta y_{\delta_{0}}^{+}\right) \times \Delta z^{+}$ & $12 \times(0.5-7) \times 10$ & $12 \times(0.5-7) \times 10$ & $12 \times(0.5-7) \times 12$ \\
\hline
\end{tabular}

\subsection{Simulation methods}

The theoretical model consists of the 3-D, compressible, Navier-Stokes equations, describing calorically perfect air with constant ratio of specific heats $(\gamma=1.4)$, constant Prandtl number $(P r=0.72)$, and temperaturedependent dynamic viscosity from Sutherland's law. The equations are solved in nondimensional, conservative form, within a well validated, massively parallel, computational framework $[1,31,34,83]$, employing structured, curvilinear grids. Spatial derivatives are computed via sixth-order, compact, central-differencing schemes [47]. Stabilizing dissipation is provided through application of an eighth-order, Padé-type $\left(\alpha_{f} \lesssim 0.45\right)$, spatial filter [32]. Shock-capturing is facilitated through local upwinding of the inviscid fluxes, in regions identified by a Ducros-like shock sensor [1]. Surrounding the shocks, the inviscid fluxes are calculated via Roe flux-differencing, from fifth-order, Weighted Essentially Non-Oscillatory (WENO), reconstructions [53], without application of the spatial filter. Time integration is achieved via an implicit Beam-Warming scheme, with time-step, $\tau \gtrsim 0.001 \delta / U_{\infty}$. In the limit of sub-iteration convergence, the scheme results in a second-order backward difference approximation for integration. The CFL number remains less than unity throughout the domain, with typical values approaching 0.1 in the outer flow and 0.7 near the wall, under which conditions the temporal accuracy of the implicit scheme is sufficient [43]. For the high-resolution, low-Reynolds-number calculations of the present work, no explicit sub-grid turbulence models are necessary [34,43]. Rather, the simulations may be described as implicit large-eddy simulation, in which the filtering procedure has been shown to effectively constitute a sub-grid model [48].

Details of the numerical discretization, boundary conditions, and mean-flow validation for the current database are well documented in previous publications $[2,4-6,8]$ and are therefore treated with brevity. The computational grids are described in Table 3 and comprise $8.9 \times 10^{7}-2.6 \times 10^{8}$ total points, with near wall resolution $\left(\Delta x^{+} \times\left(\Delta y_{\text {min }}^{+}-\Delta y_{\delta}^{+}\right) \times \Delta z^{+}\right)=(12 \times(0.5-7) \times 10)$, in which the streamwise $(x)$, platenormal $(y)$, and spanwise $(z)$ spacing are non-dimensionalized using boundary layer inner variables (denoted with "+") [73]. This resolution is sufficient for wall-resolved large-eddy simulation of both the turbulent boundary layer [61] as well as the STBLIs [52,64]. For the 3-D interactions, the span of the domain extends in excess of $17 \delta$ for all simulations to facilitate examination of 3-D flow similarity. Synthetic turbulence is introduced on the inflow boundary using a digital filtering procedure. Considerations arising from the use of modeled statistics using RANS simulations, as opposed to available simulated DNS data, and other aspects such as streamwise lengths required to establish convergence of inner and outer scales have been outlined in Adler et al. [8]. These observations are employed to choose a distance of $18 \delta$ upstream of the geometric origin to allow for development of the incoming turbulent boundary layer. The plate, ramp, and fin surfaces are modeled as adiabatic, no-slip walls. Adler and Gaitonde [5] discuss the characteristic-based boundary treatment for the other boundaries. To facilitate spectral analysis over a large frequency range, the double-fin simulation is integrated in time in excess of $1 \times 10^{4} \delta / U_{\infty}$, with temporal data resolution finer than $0.25 \delta / U_{\infty}$.

\section{Simple, swept, 3-D interactions}

An assimilation of the flow structure and dynamics of the double-fin interaction into a generalized framework requires a suitable foundation derived from simpler, swept (3-D) interactions, such as the sharp-fin and sweptcompression-ramp interactions. This follows from the observation that the double-fin interaction is formed by the merging of two opposing sharp-fin interactions; upstream of this merge region, the flow structure and spectra of the double-fin interaction may be treated as two separate sharp-fin interactions. 


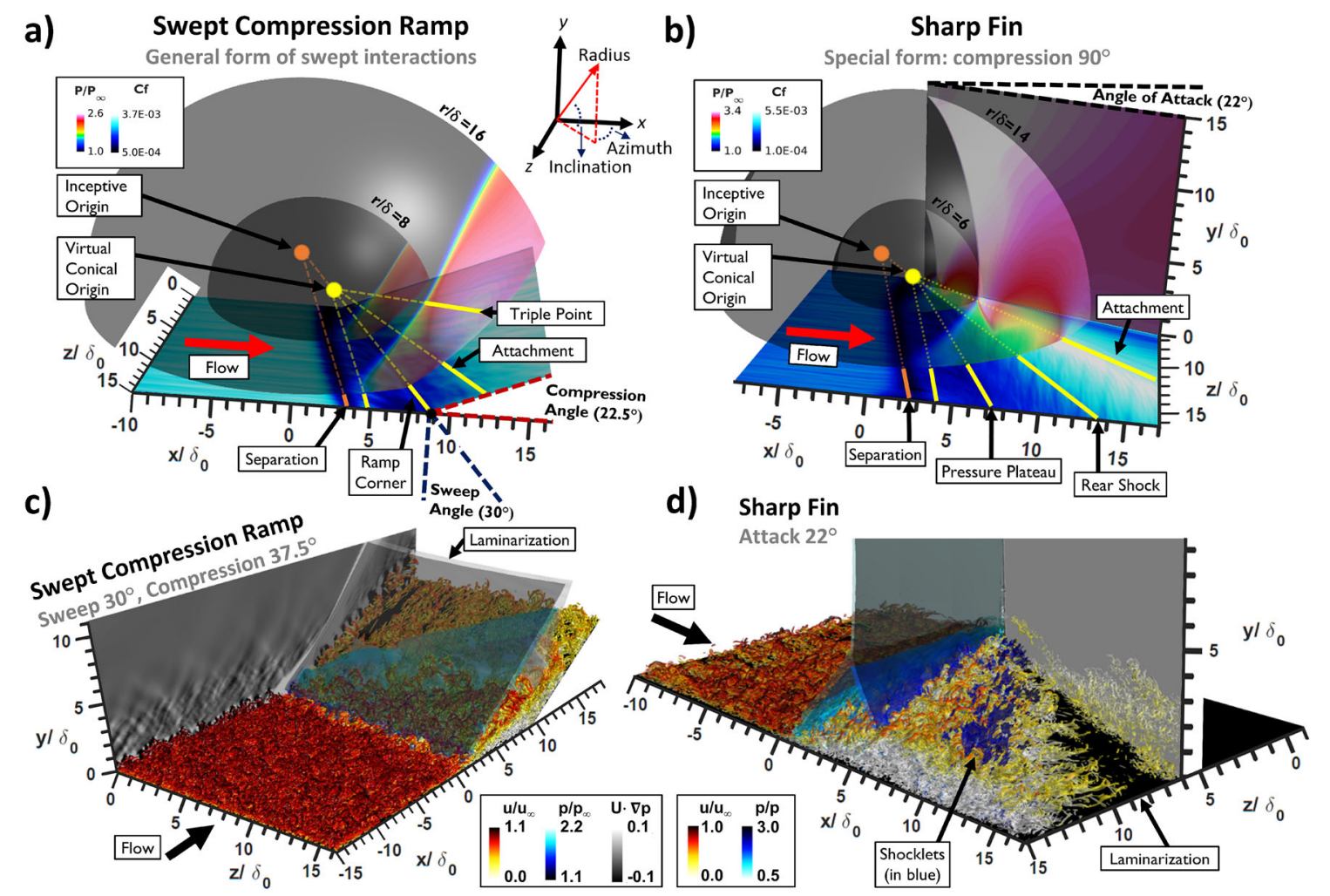

Fig. 2 Illustration of quasi-conical (spherical) mean-flow symmetry for the a SCR and b SF interactions. Concentric spherical surfaces and the fin surface are colored by pressure, whereas the plate and ramp surfaces are colored by skin-friction coefficient magnitude. Lines emanating from the virtual conical origin demonstrate conformation of the interaction features to quasi-conical symmetry, with annotations of relevant flow features included. Three-dimensional renderings of representative flow instances for the $\mathbf{c}$ SCR and $\mathbf{d}$ SF interactions. Isosurfaces of the invariant Q-criterion (colored by streamwise velocity) and shock indicated by isosurface (colored by pressure in blue) and contours of $\boldsymbol{u} \cdot \nabla p$

\subsection{Quasi-conical symmetry and free interaction theories}

Simple, swept (3-D) interactions exhibit "quasi-conical" symmetry of the mean flow. Here, "conical" describes the observation that their separation, attachment, and shock-triple-point lines outline a conical structure, with the cone apex located at the virtual conical origin (VCO). This conical structure grows linearly with distance from the VCO, introducing a 3-D scaling to the mean flow. The qualification "quasi-" indicates that the conical symmetry is not perfect. The incoming boundary layer is however, spanwise homogeneous, and thus introduces a 2-D scaling to the mean flow that is incompatible with 3-D conical symmetry. This mixing 2-D and 3-D mean flow scaling laws result in quasi-conical symmetry.

Quantitatively, the quasi-conical symmetry of these interactions is realized in spherical coordinates, in which the outer flow is self-similar and independent of the distance from the VCO. Figure 2 illustrates the quasi-conical symmetry of representative SCR (a, c) and SF (b, d) interactions. Several aspects are plotted simultaneously to highlight the relative positioning and evolution of the different aspects of interest. Frames (a) and (b) show mean pressure $\left(p / p_{\infty}\right)$ on two concentric (VCO-centered) spherical surfaces to demonstrate the radial independence of the outer flow, including the cone-like, lambda-shock structure with apex at the VCO. The skin-friction coefficient magnitude $\left(C_{f} \geq 0\right)$ is displayed on the plate and ramp surfaces to indicate the footprint of the quasi-conical outer flow on the plate surface. The angles of sweep, compression, and attack are marked, along with several mean-flow features, including the separation and attachment lines. Also marked are the VCO, as well as an inceptive origin. It is evident that the separation length ( $L$ : the arc-length between separation and attachment) grows linearly with displacement from the VCO, and unlike in the corresponding 2-D interactions, it is a local, not global, property of the flow.

Representative instances of the simple swept interactions are shown in frames (c) and (d), in which the interaction of the turbulent boundary layer (red colormap) is visualized along with the shock structure (blue 


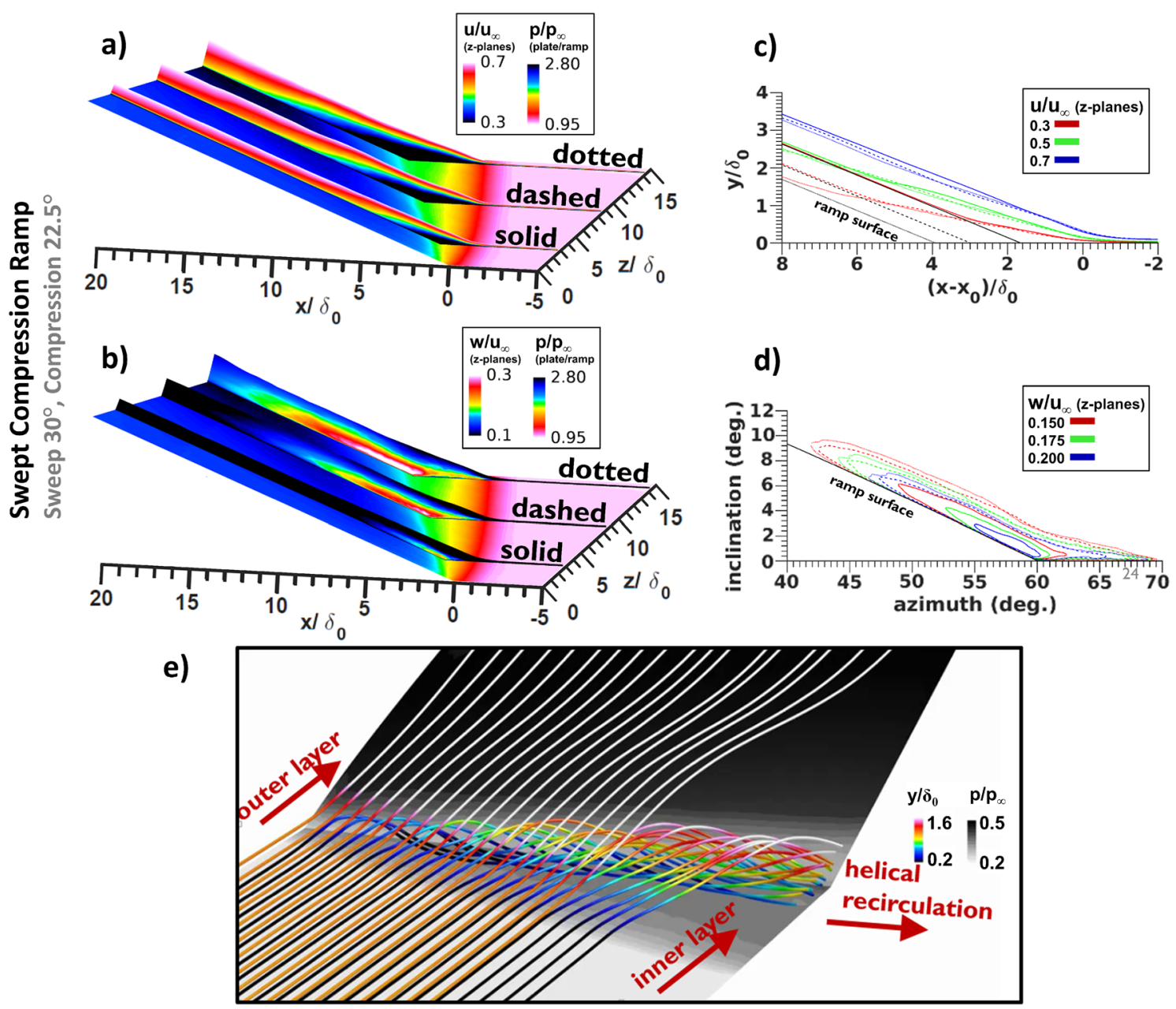

Fig. 3 Mixing of 2-D and 3-D mean-flow scaling laws in the SCR ( $30^{\circ}$ sweep, $22.5^{\circ}$ compression) interaction-evident in mean profiles of the inner (crossflow) and outer (main) shear layers at successive spanwise stations. a, b Plate and ramp surfaces colored by pressure. a Z-planes colored by streamwise velocity. b Z-planes colored by spanwise velocity. c Streamwise velocity contours from z-planes of (a); streamwise spatial coordinate aligned at local separation location. $\mathbf{d}$ Spanwise velocity contours from z-planes of (b) in spherical VCO-centered coordinates. e Streamlines of the mean flow associated with the inner and outer shear layers reflect the 2-D character of the outer layer and the 3-D character of the inner layer; streamlines of the inner layer trace a helix in the crossflow direction

colormap), highlighting the large separation of scales between the shock structure and turbulent fluctuations. The quasi-conical symmetry is evident in the lambda-shock structure and outer flow of both interactions, as well as in the footprint of this outer flow on the boundary layer turbulence; this includes lines of separation and attachment, as well as post-separation growth of the separated shear layer. Downstream of attachment, a significant reduction in turbulence kinetic energy occurs in both interactions due to similarities in the 3-D attachment process. However, there are differences between the SCR and SF interactions downstream of the main shock because attachment for the SCR interaction occurs on the shock generator (ramp) surface, whereas attachment for the SF interaction occurs on the plate surface.

The ramifications of the mixing 2-D and 3-D scaling laws of the simple swept interactions are further explored in Fig. 3. For brevity, the discussion here is focused on the SCR interaction; however, the same principles hold for the SF [7], and the DF interaction discussed below. The plate and ramp surfaces (a, b) are colored by mean pressure, reflecting the quasi-conical footprint of the interaction, whereas the outer (main) shear layer is isolated by z-plane contours of mean streamwise velocity (a) at several spanwise stations, and the inner (crossflow) shear layer is isolated by z-plane contours of mean spanwise velocity (b) at several spanwise stations. Frames (a) and (c) quantitatively demonstrate the 2-D (spanwise-homogeneous) similarity of the outer shear layer, supporting the claim of classical (2-D) free-interaction theory of Chapman et al. [18], 
in that the mean flow profiles in the vicinity of separation are independent of the downstream obstacle (which becomes larger in the spanwise direction); the streamwise velocity contours associated with the outer shear layer collapse well in Cartesian coordinates (c) offset by the local separation location. Frames (b) and (d) quantitatively demonstrate the 3-D (conical) symmetry of the inner shear layer, supporting the claim of conical (3-D) free-interaction theory of Settles and Kimmel [72], in that, for given upstream conditions and sweptshock geometry, the mean flow profiles in the quasi-conical interaction region exhibit collapse in spherical (VCO-centered) coordinates; the spanwise velocity contours associated with the inner shear layer collapse well in spherical coordinates (d) with origin at the VCO. Frame (e) includes representative streamlines of both the inner and outer shear layers, reflecting the 2-D character of the outer shear layer, which exhibits minimal spanwise deflection, as well as the 3-D character of the inner shear layer, which exhibits a significant crossflow component and results in a helical pattern. Here, the plate and ramp surfaces are colored by mean pressure, whereas the streamlines are colored by distance from the plate.

\subsection{Connections between swept mean flow 2-D and 3-D scaling laws and associated spectra}

The interplay between 2-D and 3-D scaling laws in the mean flow profiles of the post-separation shear layers has ramifications on the development of coherent fluctuations and resulting flow spectra. The major result is that mid-frequency fluctuations in these shear layers also contain the signatures of the mixing of 2-D and 3-D scaling laws, suggesting that the prominent mid-frequency band of unsteadiness depends on the spatially localized flow structure, and in particular, that the frequencies of this band depend on the local length scales of the shear layers. A relationship between local shear layer properties and frequency scales is also identified in 2-D STBLIs [41]. Adler and Gaitonde [7] discuss in detail the mid-frequency spectral scaling properties and associated coherent structures for a variety of simple, swept, 3-D interactions, including both SF and SCR interactions, which exhibit similar properties.

Briefly, the relationship of the spatial signature of the wall-pressure power spectral density (PSD) to the interaction strength and radial displacement from the VCO can provide a connection between different frequency ranges and corresponding flow scales. Specifically, this relationship is exploited to identify the 2-D and 3-D scaling-laws present in the spectra, along with the spatial signature indicating where these scaling laws are relevant. Considering swept-compression-ramp interactions as an example, the wall-pressure PSD is shown in Fig. 4. The PSD is weighted by frequency for proper representation on a logarithmic scale and normalized independently at each spatial location to focus on the spatial distribution of frequency independent of the magnitude of fluctuations. The PSD is computed using Welch's windowed overlapping segments estimator method by splitting the signal into 8 segments with $50 \%$ overlap using Hamming windows. The plots are arranged in a matrix; going left-to-right across the rows increases radius from the VCO, while top-to-bottom down the columns increases interaction strength. A conceptual understanding of interaction scaling is facilitated by discerning patterns in the data across different interaction strengths and radii. Identifying locations where the PSD signature displays qualitatively perceptible changes in behavior aids in understanding the scaling. The blue lines connecting the plots in columns facilitate comparison of the spatial scaling of the surface fluctuations for different interaction strengths, while red lines aid in the corresponding comparison of frequency scaling for different radii. Divergence of blue lines with increasing interaction strength reflects the increase in interaction spreading rate with increasing interaction strength; that is, the arc-length between separation and attachment grows more rapidly with increasing radius for stronger interactions. Considering the red lines, horizontal alignment reflects 2-D frequency scaling (scaling with $S t_{\delta}$ on the left axis), which is especially applicable for the high-frequency surface fluctuations $\left(S t_{\delta} \gtrsim 0.2\right)$. With Strouhal number based on the local separation arclength $\left(S t_{L}\right)$ on the right axis, the diverging red lines thus reflect the competing 2-D (horizontal with constant $S t_{\delta}$ ) and 3-D (downward sloping with constant $S t_{L}$ ) scaling exhibited by the mid-frequency fluctuations. Similar results hold for the sharp-fin interactions [7].

Upstream of the interaction region, the prominent high-frequencies are typical of coherent integral-scale features of the incoming turbulent boundary layer $\left(S t_{\delta} \sim 1\right)$. These are modulated throughout the interaction, monotonically decreasing through the pre-separation, interaction, and post-attachment zones, as the boundary layer grows and slows; however, these frequencies are essentially independent of the local separation length (comparing along the rows), and are relatively independent of the interaction strength (comparing along the columns). The exception is differences in the separation and attachment locations, which depend on the interaction strength. Mid-frequency content $\left(0.01 \lesssim S t_{\delta} \lesssim 0.2,0.1 \lesssim S t_{L} \lesssim 1\right)$ is present between the separation and attachment locations; these correspond to coherent Kelvin-Helmholtz-like (K-H) shear-layer 


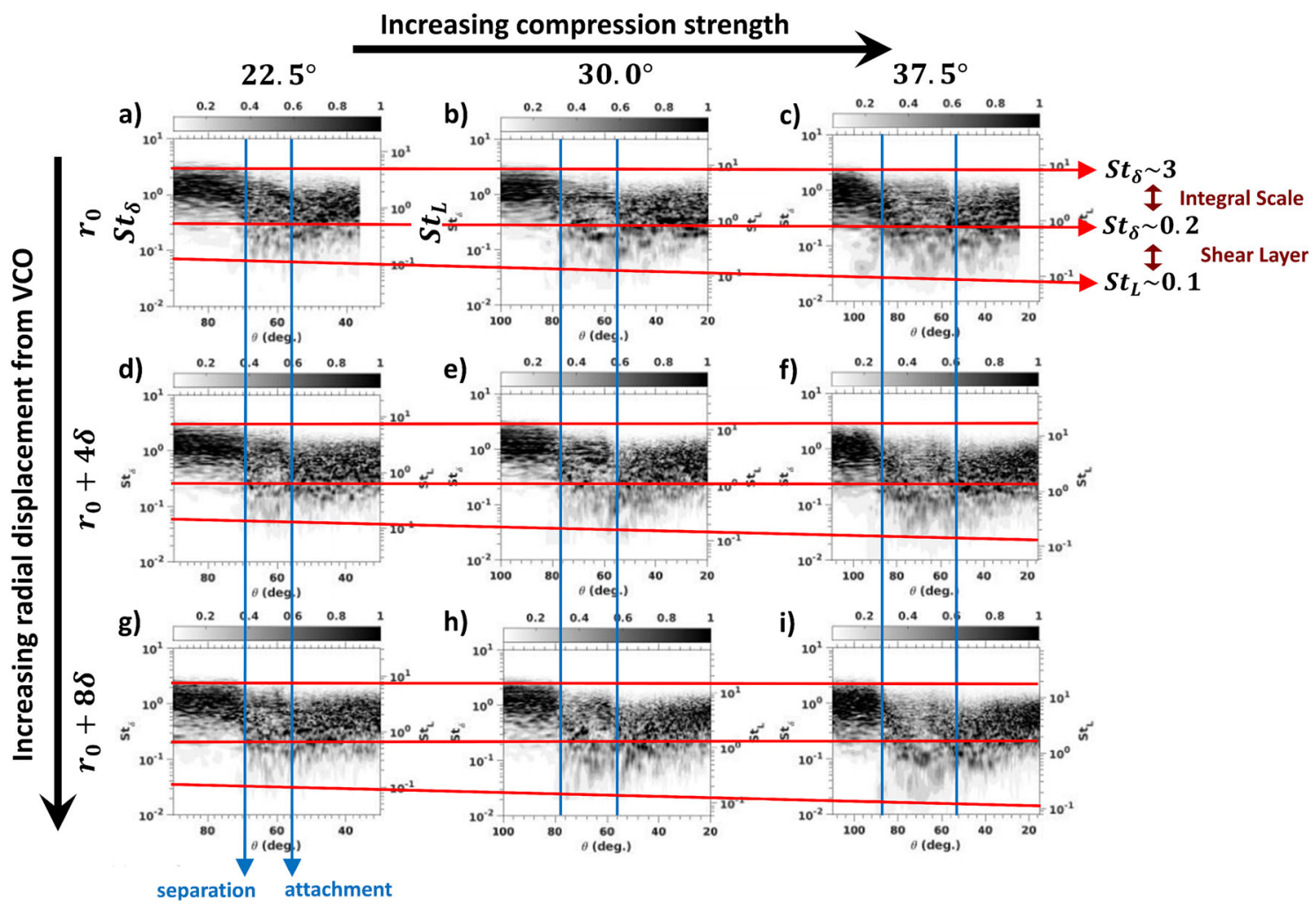

Fig. 4 Mixing of 2-D and 3-D scaling laws for mid-frequency unsteadiness in SCR interactions: Premultiplied and normalized fluctuating wall-pressure power spectral density (PSD) as a function of frequency (vertical axis) and azimuthal angle (horizontal axis-describing the spatial location: $\theta=0$ is the freestream direction). Frequency is described by boundary layer Strouhal number $\left(S t_{\delta}\right.$ : left vertical) and local separation arc-length Strouhal number ( $S t_{L}$ : right vertical). The PSD matrix is organized via increasing radial displacement from the VCO left-to-right across the rows and increasing compression strength top-to-bottom down the columns to demonstrate the competing 2-D scaling (horizontal red lines) and 3-D scaling (downward sloping read lines) for the mid-frequency unsteadiness. Additionally, the increase of interaction spreading rate with interaction strength is demonstrated by the non-parallel blue lines (color figure online)

phenomena. The relationship between this coherent content to that in spanwise-homogeneous interactions [3, 10,13] has been discussed in Adler and Gaitonde [7] The behavior of this band differs in 3-D interactions due to the differences in the post-separation shear layers: the outer (main) shear layer with spanwise-homogeneous (2-D) scaling, and the inner (crossflow) shear layer with conical (3-D) scaling. In both SCR and SF (and early DF stages, which resemble SF), this mid-frequency unsteadiness demonstrates a linear dependence on the local separation length (demonstrated by the downward sloping red line forming the lower bound of the midfrequency range); i.e. $S t_{L}$ (3-D scaling) is more appropriate than $S t_{\delta}$ (2-D scaling) for describing this lower bound of the mid-frequency content. Characteristics of the development of coherent structures associated with this mid-frequency band in simple swept interactions are discussed by Adler and Gaitonde [7].

\section{Compound, 3-D, double-fin interaction}

We now examine the double-fin inlet/isolator interaction, which exhibits several features that require an adjustment of flow structure and dynamical description relative to simple, swept (3-D) interactions. The changes in the separation structure due to the merging of the opposing sharp-fin interactions are described first, followed by a discussion of the unsteadiness characteristics. 


\subsection{Flow structure}

Figure 5 describes several key features of the flow structure, highlighting the closure of the primary separation and initiation of secondary separation, sidewall interaction and complex corner flow in the isolator section. Frame (a) includes a 3-D rendering of a representative instance of the flow, with the turbulent structure rendered in the red colormap and the shock structure rendered in the blue colormap. Two opposing sharp-fin interactions are generated at the point where the incoming boundary layer encounters the fin leading edges at the start of the inlet. These develop downstream relatively independently until the effects of interaction merging become significant in the region of the shock crossing, forming a compound 3-D interaction. Shortly after the shock crossing, the sidewall-compression inlet transitions to the rectangular constant cross-sectional area isolator section in accordance with the corresponding experiment. Quasi-2-D interactions are formed on the sidewalls where the shocks impinge on the sidewalls of the isolator. Frame (b) displays surface flow features associated at the walls; the annotations highlight the different interactions that comprise the compound interaction. There is similarity with the sharp-fin observations in the initial region. The fin surface shows quasi-2-D interactions with features similar to 2-D interactions with sidewall confinement effects. The surface-flow lines in the region of the compound 3-D interaction near the center of the channel are unique to double-fin-like interactions. Generally, the surface-flow lines may be used to infer separation (converging) or attachment (diverging) lines of the flow. The primary separation and attachment lines may be identified in this way; however, inferring the presence of secondary separation or attachment features from converging or diverging lines is less robust due to the compressible character of this flow, and more detailed confirmation must be obtained from the off-surface flow structure (for example, 3-D streamlines).

Details of the compound 3-D interaction are described by frames (c-e). Frame (c) shows surface-flow lines in the central region of the plate, colored by skin-friction-coefficient magnitude. The annotations identify the primary separation and primary attachment lines (associated with the simple 3-D interactions) as well as the location of primary separation closure and secondary separation associated with the compound interaction. Frame (d) provides a close-up of the primary separation closure region, where the primary separation lines terminate at a separation node (followed by an attachment saddle). The flow separation angle at the nodal termination point on the symmetry plane is much larger than away from it because of the more rapid pressure rise associated with the crossing-shocks. Frame (e) highlights the region of secondary separation, where the previously attached flow encounters a separation-node/attachment-saddle pair and also separates rapidly from the plate surface.

The structure of flow associated with the region of the quasi-2-D sidewall interactions is highlighted in frames (f, g). Frame (f) shows surface-flow lines in the region of the compound 3-D interaction with clearly evident lines of separation and attachment; the sidewall itself is colored by skin-friction-coefficient magnitude. Frame (g) describes this region from a different viewpoint, in which the separation saddle and attachment node of the quasi-2-D interaction are evident. Surface-flow lines of the plate surface (purple) are also included to show the complex flow developing in the isolator corner in the region of the sidewall interaction. Separation and attachment phenomena on both the plate and sidewall surfaces further complicate the flow structure and dynamics of the isolator section. Some quantitative descriptions of these mean flow features may be found in Adler and Gaitonde [6].

\subsection{Band-isolated dynamics}

A detailed analysis of the unsteadiness in the DF configuration indicates the presence of four prominent dynamically significant frequency bands. A convenient frequency $(f)$ delineation is based on Strouhal number $\left(S t_{\delta}=f \delta / U_{\infty}\right)$ formed from the freestream velocity $\left(U_{\infty}\right)$ and incoming boundary layer thickness $(\delta)$. With this scaling, the flowfield displays very-high-frequency (fine-scale turbulence: $2 \lesssim S t_{\delta}$ ), highfrequency (integral-scale turbulence: $0.2 \lesssim S t_{\delta} \lesssim 2$ )), mid-frequency (coherent shear-layer fluctuations: $0.02 \lesssim S t_{\delta} \lesssim 0.2$ ), and low-frequency (interaction-"breathing" phenomena: $S t_{\delta} \lesssim 0.02$ ) bands. Using the method of Adler and Gaitonde [3], we decompose the pressure fluctuations into four frequency bands, using a time-domain filtering procedure, resulting in a complete basis describing pressure fluctuations. Representative instances of each of these bands are described in Fig. 6, which plots band-isolated pressure fluctuations on the interaction centerline, and Fig. 7, describing band-isolated pressure fluctuations on a plane parallel to the plate surface at height $y / \delta=3.5$. The overlaid black lines provide a reference to relate the pressure fluctuations to the mean flow structure, including the main shocks and expansions as they cross the planes. Frame (a) extracts 

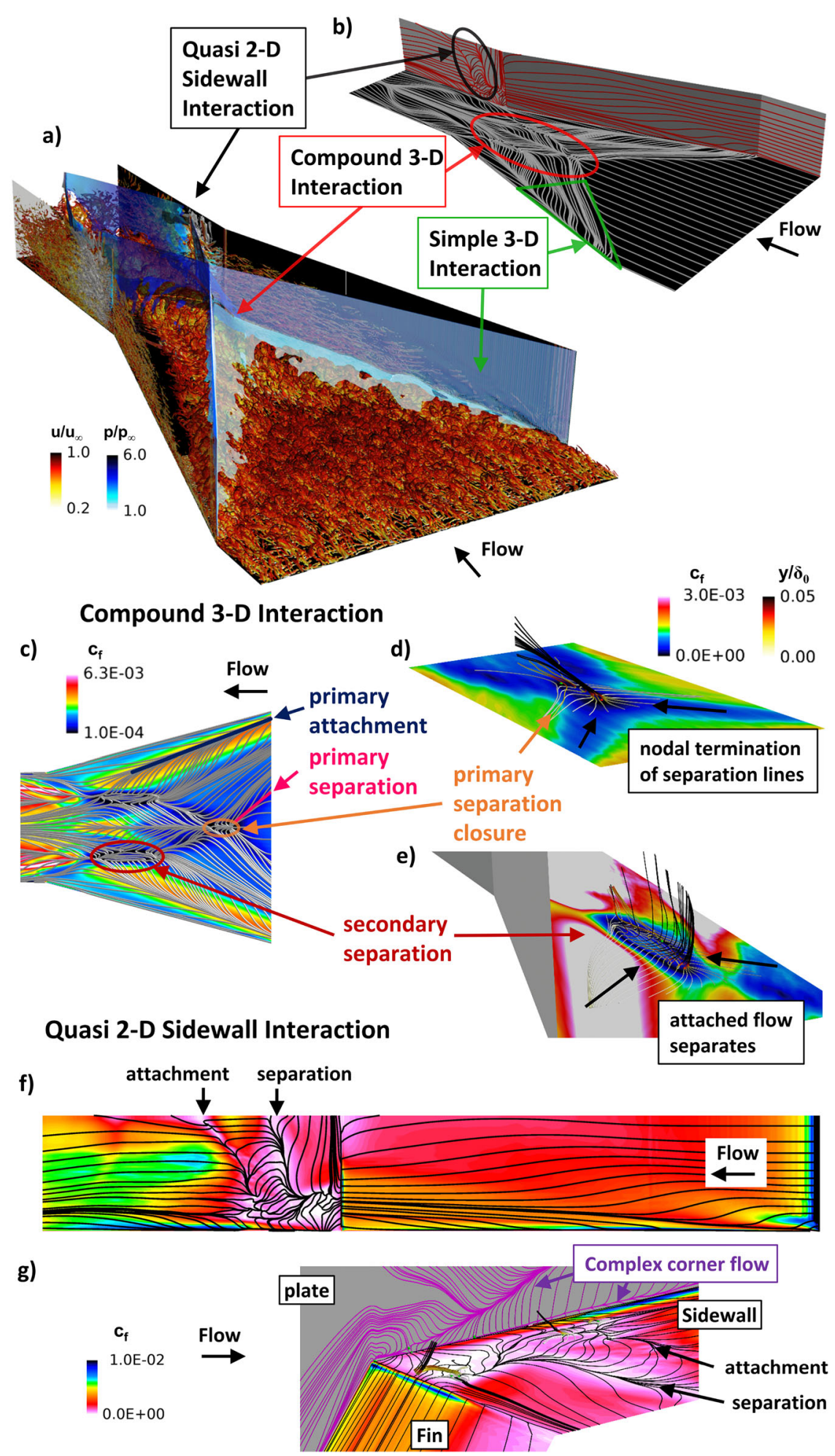

Fig. 5 Flow structure of DF interaction. a Isosurfaces of Q-criterion (colored by streamwise velocity). Shocks as in Fig. 2. b Surface flow visualization indicating separation and attachment lines on the plate (black) and side (gray) walls with annotations highlighting the comprising interaction regions. c-e Compound 3-D interaction features. c Surface flow visualization on plate colored by skin-friction coefficient magnitude highlighting important flow features $(\mathbf{d})$ and secondary separation $(\mathbf{e})$ regions. $\mathbf{f}, \mathbf{g}$ Quasi-2-D sidewall interaction features. f Surface flow visualization on sidewall colored by skin-friction coefficient. Annotations highlight important flow features. $\mathrm{g}$ Details of sidewall separation and attachment with complex corner flow 


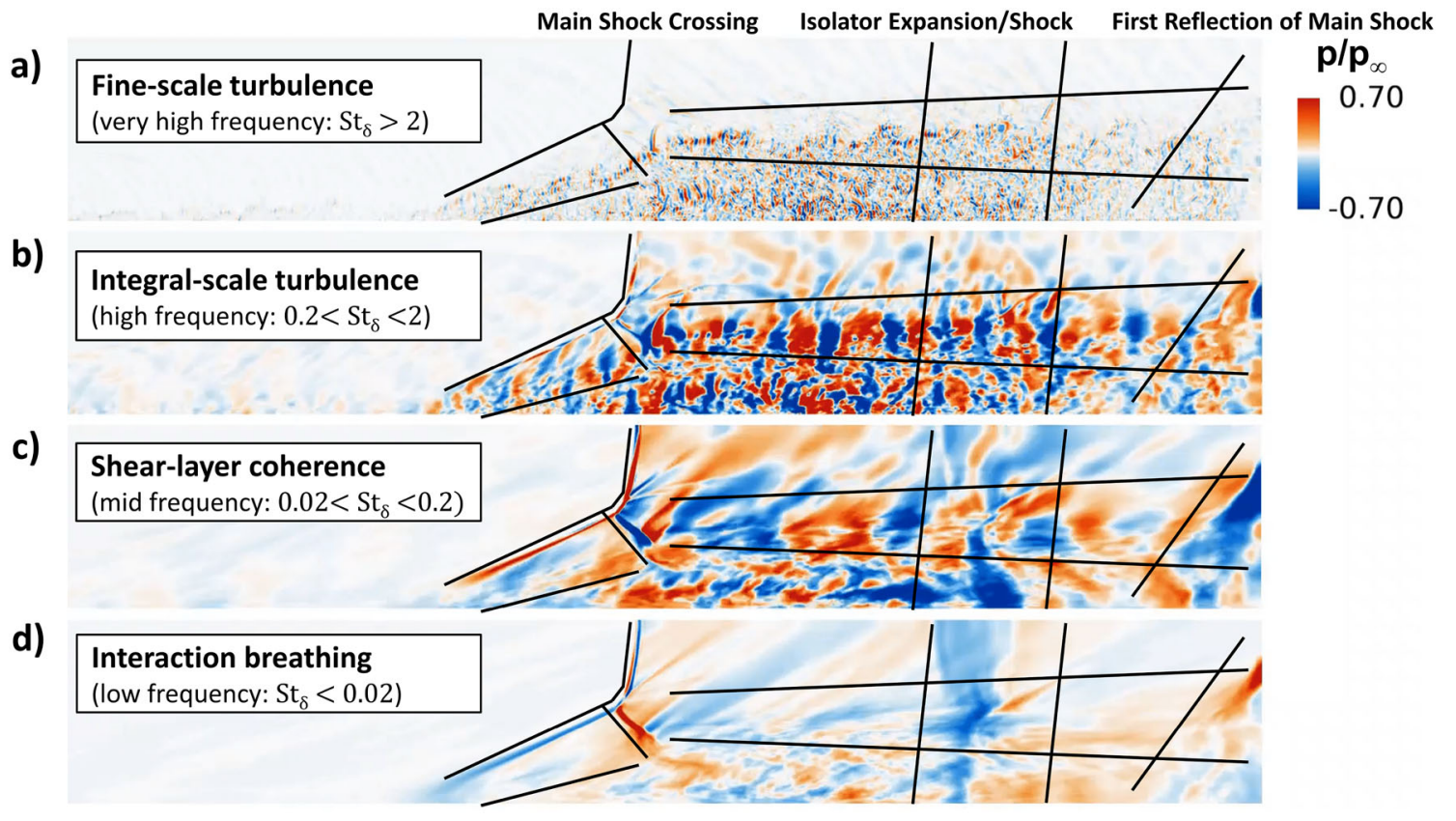

Fig. 6 Band-isolated pressure fluctuations (normalized by freestream pressure) along the centerline (spanwise normal plane) of the DF interaction with annotative black lines to provide reference positions for various structural features. A broad spectrum of unsteadiness is exhibited by the compound, 3-D STBLI, delineated into four primary frequency bands. a Very high frequency $\left(2 \lesssim S t_{\delta}\right)$. b High frequency $\left(0.2 \lesssim S t_{\delta} \lesssim 2\right)$. c Mid-frequency $\left(0.02 \lesssim S t_{\delta} \lesssim 0.2\right)$. d Low frequency $\left(S t_{\delta} \lesssim 0.02\right)$

the very-high-frequency band associated with fine-scale turbulent structures; this band is primarily convective in character and has relatively little influence on shock rippling. Frame (b) describes the high-frequency band associated with integral-scale turbulent structures; this band is also primarily convective in character and influences shock rippling in a very localized manner. Frame (c) describes the mid-frequency band associated with shear-layer coherent structures; this band is also primarily convective in character and also influences shock rippling, but in a less localized manner. Frame (d) describes the low-frequency band associated with the large-scale interaction "breathing"; this band is primarily oscillatory in character and influences shock rippling in a non-local manner, indicating that the shock structure as a whole is actively oscillating in this frequency range. Note the relative insignificance of this low-frequency band upstream of the compound 3-D interaction. The encompassing trend moving toward lower frequency involves the transition from high-frequency, convective fluctuations, with predominantly local influence on the shock structure to low-frequency, oscillatory fluctuations, with predominantly non-local influence on the shock structure. Overall, after the shock crossing, the high-frequency bands have relatively small influence on the shock structure compared to the low-frequency band.

\section{Influence of separation structure on dynamics}

This section illustrates some of the important dynamical differences in terms of wall-pressure spectra and then presents several arguments to understand how changes in the structure of the mean flow lead to these differences in the dynamics. The low-frequency range of unsteadiness shows notable differences among the different interaction classes: spanwise-homogeneous-2-D, simple-swept-3-D, and compound-3-D interactions. The low-frequency range refers to the range characterized by the features of frames (d) of Figs. 6 and 7, exhibiting oscillatory (not convective) and relatively non-local and coherent fluctuations of the shock structure. This behavior is typical of the lowest significant band of frequencies in each flow. The characterization of this band as "low-frequency" is relative, because the absolute frequency of each band corresponds to appropriate flow velocity and length scales leading to the establishment of the mechanism of unsteadiness. For instance, the shear layer phenomena underlying the dynamics of the mid-frequency band may result in low frequencies in absolute terms if the size of the 3-D separation becomes large [7]. 


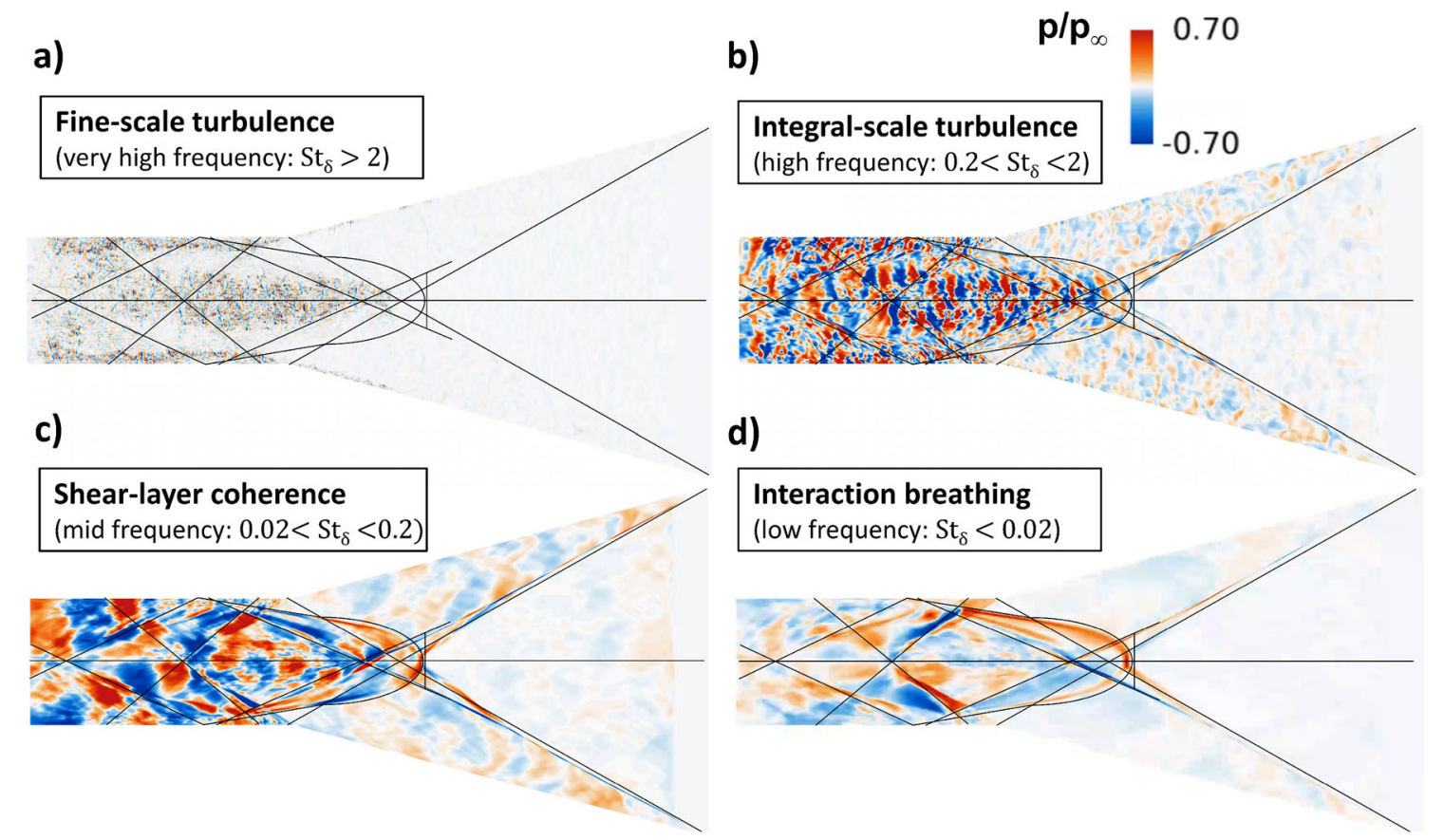

Fig. 7 Band-isolated pressure fluctuations (normalized by freestream pressure) along a plate-normal plane, above the incoming boundary layer $(y / \delta=3.5)$, of the DF interaction with annotative black lines to provide reference positions for various structural features. A broad spectrum of unsteadiness is exhibited by the compound, 3-D STBLI, delineated into four primary frequency bands. a Very high frequency: $\left(2 \lesssim S t_{\delta}\right)$. b High frequency $\left(0.2 \lesssim S t_{\delta} \lesssim 2\right)$. c Mid-frequency $\left(0.02 \lesssim S t_{\delta} \lesssim 0.2\right)$. d Low frequency $\left(S t_{\delta} \lesssim 0.02\right)$

The 3-D nature of the flow poses a significant challenge in establishing the connections between the spectra associated with critical regions of the flowfield. To address this, we present a composite in Fig. 8, which shows frequency-weighted wall-pressure spectra at different locations for each flowfield of interest. In each case, a representative instantaneous snapshot is provided, frames $(\mathrm{a}-\mathrm{c})$ and $(\mathrm{g})$ with arrows and annotations connecting important features to the corresponding spectra $(\mathrm{d}-\mathrm{f}, \mathrm{h}-\mathrm{j})$. In the spectral plots, the vertical axis represents frequency while the horizontal axis traverses the spatial extent as specified individually for each interaction. The spectra have been independently normalized at each spatial location to highlight the spatial distribution of prominent frequencies on the same scale. For the IS (a, d), SCR (b, e), and SF (c, f) interactions, the flow separation and attachment locations are marked, and several important frequency bands are highlighted on the spectra. Here we employ the 2-D impinging shock interaction of [3] for comparison; the same result holds for the 2-D compression ramp interaction of [7]. The spatial coordinate corresponds to the streamwise distance normalized by incoming boundary layer thickness for the IS and DF interactions, whereas the spatial coordinate corresponds to the azimuthal angle (with constant radius) for the SCR and SF interactions. The principal frequency bands include: (1) the high-frequency band with orange annotation describing the fine and integral scale turbulence, (2) the mid-frequency band with green annotation describing the separated shear layer coherence and shedding, (3) the low-frequency separation bubble oscillations with red annotation, and (4) the low-frequency separation breathing with purple annotation. Notably, these lower frequency bands are significantly muted in the simple-swept-3-D interactions relative to the same bands in spanwise-homogeneous2-D interactions. Adler and Gaitonde [7] provide additional analysis and discussion relevant to this point, including comparisons between swept and unswept compression-ramp interactions with identical upstream conditions that succinctly demonstrate this phenomenon.

For the DF interaction $(\mathrm{g}-\mathrm{j})$, the three spectra are taken along lines following the sidewall/plate corner (h), the quarter-span line (i), and the half-span line (j). Here, prominent regions of the spectra are connected to the regions of the flow rendering, with grouping based on the sub-interaction class. Green annotations connect regions of the spectra corresponding to the SF interactions upstream of interaction merging to the flow rendering. The dynamical and mean flow properties in this region are similar between the DF and SF interaction, and the DF spectra are characterized by prominent high- and mid-frequency bands in the same 


\section{2-D: Impinging Shock}

a)

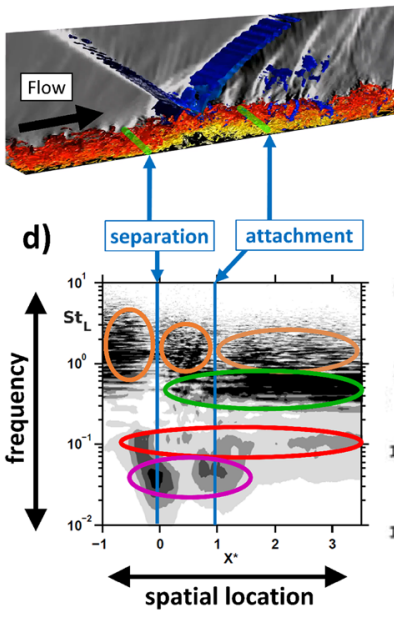

Prominent frequency bands and associated flow features

\section{3-D: Swept Compression Ramp}

\section{3-D: Sharp Fin}

b)

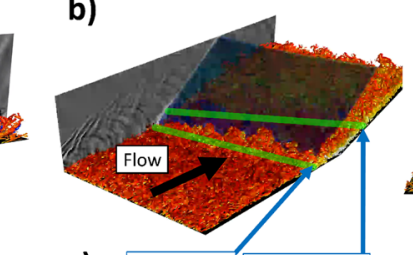

e)

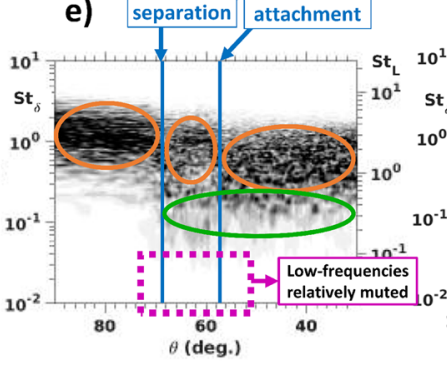

f) separation attachment

c)
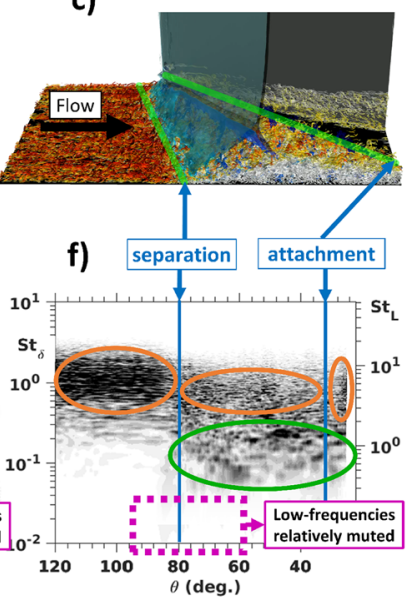

$f \times P S D(f)$

$\begin{array}{lllll}0.2 & 0.4 & 0.6 & 0.8 & 1\end{array}$

Frequency weighted wall pressure power spectral density normalized at each spatial location

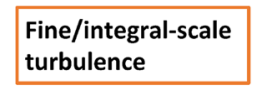

\section{Separated shear layer coherence and shedding}

\section{Separation bubble} oscillations
Low-frequency separation breathing and shock motion

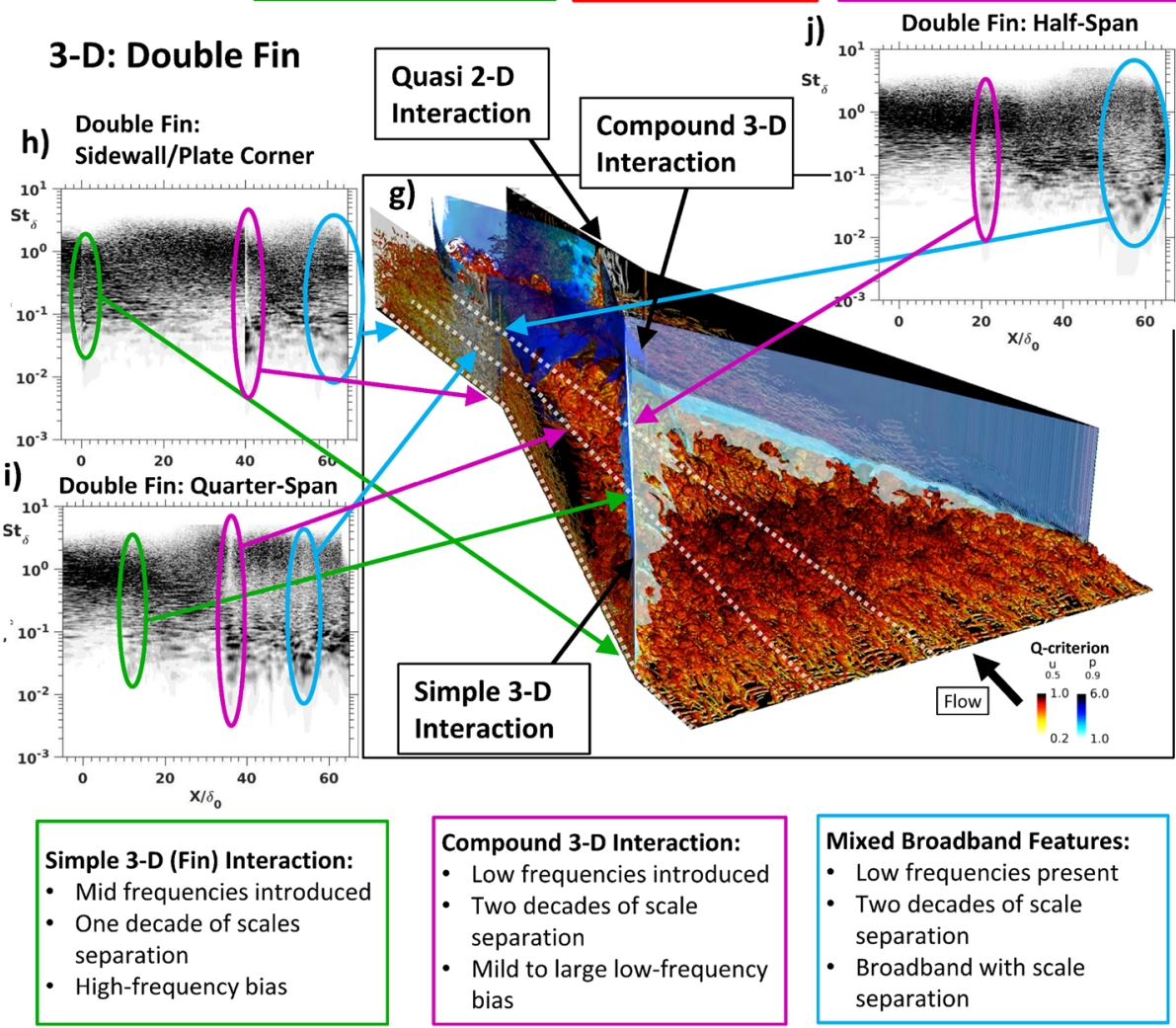

Fig. 8 Comparison of spectra between various interaction types. a-c Representative 2-D and simple 3-D interactions with annotated separation and attachment lines. d-f Frequency weighted wall-pressure PSD as a function of frequency and spatial location (streamwise distance (d); azimuthal angle $(\mathbf{e}, \mathbf{f})$ ). Wall-pressure PSD as a function of streamwise distance along the sidewall/plate-corner (h), quarter-span (i), and half-span (j) lines of the DF interaction, with annotations showing corresponding flow features $(\mathbf{g})$ 


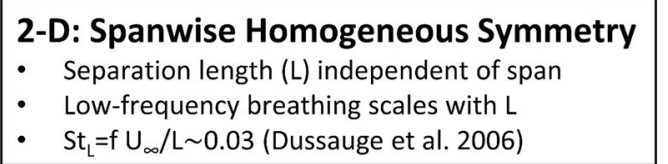

a)

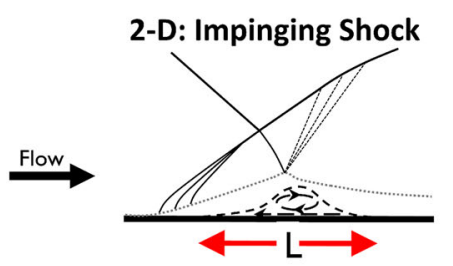

\section{3-D: Quasi-Conical (Spherical) Symmetry}

- Separation (arc) length (L) grows linearly with span

- $\mathrm{St}_{L} \sim 0.1$ relevant to shear layer dynamics

- $\mathrm{St}_{\mathrm{L}} \sim 0.03$ not relevant to separation breathing

b)

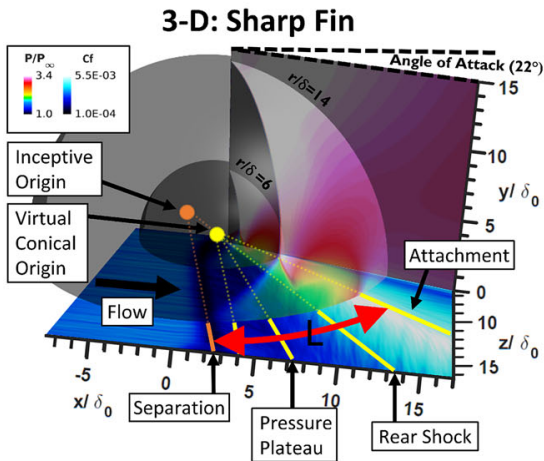

d)
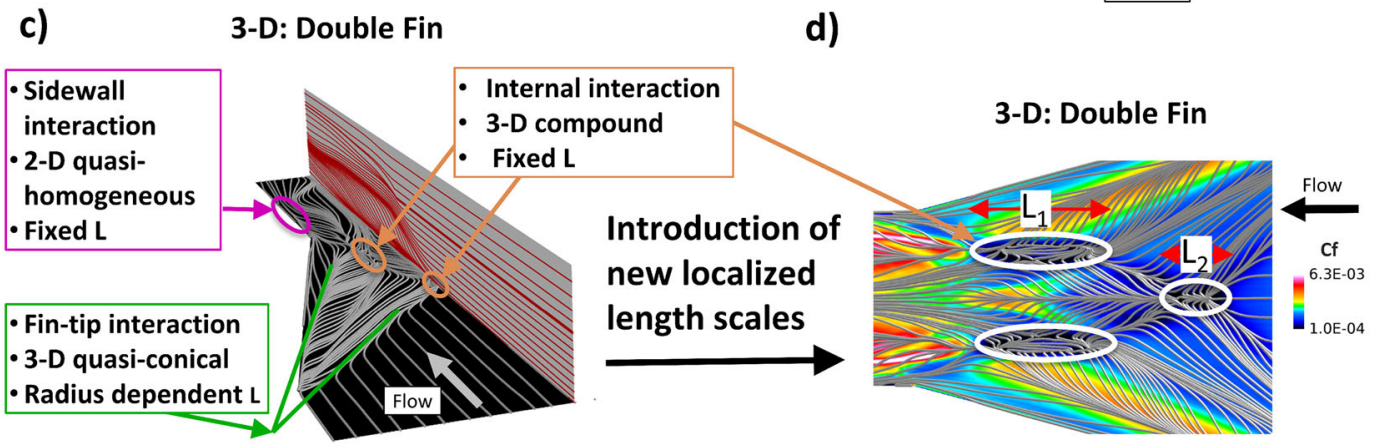

Fig. 9 Illustration of flow symmetry and separation length scales. a Representative 2-D interaction with annotated separation length (spanwise homogeneous symmetry, global separation length scale with established relationship to low-frequency unsteadiness). b Representative simple, swept, 3-D interaction with annotated radially-dependent separation length (quasi-conical symmetry, spatially dependent separation length with relationship to mid-frequency unsteadiness). c, d Representative compound, swept, 3-D interaction (complex symmetry, both fixed and spatially dependent separation length scales with established relationship to mid-frequency unsteadiness and possible relationship to low-frequency unsteadiness). c, d Annotations highlight the regions with new separation length scales in the DF interaction

way as the SF interaction. Purple annotations connect regions of the spectra corresponding to the compound $3-\mathrm{D}$ interaction in the region of shock crossing and interaction merging to the flow rendering. These spectra are unique to the DF interaction, and are characterized by significant low-frequency biases in the spectra, more similar to 2-D interactions than simple 3-D interactions. Blue annotations connect regions of the spectra in the isolator duct; these regions correspond to the quasi-2-D-sidewall interaction as well as persisting unsteadiness in the shock structure resulting from the upstream compound 3-D interaction. The unsteadiness in this region is quite broadband, and even more complex, due to the compounding of sources as the flow moves downstream. In the isolator, certain regions demonstrate a significant low-frequency bias (i) whereas others exhibit more of a bi-modal broadband distribution (j). The crucial observation from these spectra is that regions with significant low-frequency bias develop in the region of the compound interaction, that were not present in the $\mathrm{SF}$ interactions before interaction merging.

The question now arises: How can these differences in the dynamical properties of the interactions be understood from differences in the mean flow structure? It would be desirable to understand how regions of significant low-frequency bias in the spectra could be deduced from fundamental principles, without the need for intensive simulations or experiments. Several arguments are discussed below, which are relevant to but not exhaustive of the structure/dynamics relationships in complex STBLIs.

The first argument is illustrated in Fig. 9, relating to the length scales of the separation. As discussed by Dussauge et al. [25] (among others), a relationship exists between the mean separation length $(L)$ and the prominent low-frequency unsteadiness, with $S t_{L} \sim 0.03$ describing the low-frequency band over a wide range of flow parameters. This is illustrated in frame (a) for the IS interaction, in which the separation length is 
independent of span (a global length scale for all 2-D interactions). For simple-swept-3-D interactions, the separation length (interpreted here as the VCO-centered arc-length between the separation and attachment lines) is a local (not global) property of the flow; therefore, any spectral phenomena scaling with this length scale must be local (not global) phenomena. Observation of the simple 3-D interactions suggests that $S t_{L} \sim 0.1$ is an important scale for local shear-layer phenomena (mid-frequency fluctuations), but $S t_{L} \sim 0.03$ does not appear to be relevant for global interaction breathing (low-frequency fluctuations). This is illustrated in frame (b) for the SF interaction, in which the separation length grows linearly with distance from the VCO (a local length scale for all simple 3-D interactions).

This contrasts with the DF configuration, which exhibits three interaction types, each with different length scales. The simple 3-D interactions (upstream of interaction merging) exhibit a local length scale similar to the $\mathrm{SF}$ interaction that depends on the distance from the VCO. The quasi-2-D interaction on the side wall exhibits an essentially global separation length like that of 2-D interactions with sidewall confinement effects. However, in the compound 3-D interaction region of the DF flow, new length scales emerge, associated with the closure of the primary separation and the initiation of the secondary separation. Frame (c) highlights the regions of the DF surface flow in which the three classes of interactions are observed, with annotations describing the properties of the separation length in each region. Frame (d) focuses on the plate surface flow indicating the relevant length scales of separation for the compound 3-D interaction. These length scales contrast with both those of the 2-D interaction and simple 3-D interaction, in that they do not describe the distance between lines of separation and attachment; rather, they describe the distance between the surface-flow singular points. It may be that these separation length scales of the compound 3-D interaction are important to the establishment of relevant frequency scales; however, verification of this relationship requires an extensive parametric study of different compound 3-D interactions, in conjunction with an exhaustive experimental campaign.

The second argument, illustrated in Fig. 10, relates to the structure of the separation. Describing separation and attachment in highly-three-dimensional flows is a challenging task. For 3-D separation, streamlines of the separating flow do not necessarily reattach [30], and the separation surface topology is often more suitably described in terms of singular points [19], as opposed to global lines of separation and attachment, which are the norm in 2-D. The singular point description of separation and attachment for a representative spanwisehomogeneous 2-D interaction is illustrated in frames (a, b). Here, the lines of separation and attachment may be decomposed into singular-point pairs, which are degenerate across the span, in which a pair of one type is followed by a pair of the opposite type across the span. For instance, the 2-D separation will be initiated by a separation saddle and the 2-D attachment will be initiated by an attachment node. Along the separation line, separation saddles and separation nodes alternate across the span, while along the attachment line, attachment nodes and attachment saddles alternate across the span. Therefore, the separation length in 2-D interactions represents the distance between the initial separation-saddle and attachment-node singular points - the commonly used separation length in 2-D interactions describes the distance between surface-flow singular points. These considerations follow those of Green [37] for 2-D STBLIs and Chapman and Yates [19] for singular-point descriptions of general 3-D separation and attachment phenomena.

This contrasts with simple-swept-3-D interactions, in which only a single pair of singular points is observed in the surface flow (providing the interaction is infinite); of course, termination of the interaction by means of interaction merging in the DF interaction, or an expansion wave in a semi-infinite simple 3-D interaction, will break the quasi-conical symmetry and change the separation structure outside of the region of the flow that can be approximated as an infinite interaction. Figure 10 frame (c) illustrates the separation-saddle and attachmentnode singular points in the surface flow of the sharp fin interaction; these points occur very near to the fin leading edge because the fin has no bluntness, and they initiate the separation and attachment lines, respectively. Frame (d) again shows the streamlines of the inner and outer shear layers of the swept-compression-ramp interaction. The initiation of separation and attachment in this interaction is similar to the sharp-fin interaction, and is located near the upstream ramp-corner/sidewall junction. If the helical streamlines of the inner layer were to continue indefinitely across the span, no additional singular points could occur in the surface flow. In this sense, the separation in simple-swept-3-D interaction remains topologically open. Therefore, unlike the 2-D interaction, the commonly discussed separation length in the simple 3-D interactions does not represent the distance between the separation-saddle and attachment-node singular points found near the origin of the interaction.

The relative association between the different singular points is more complicated for the DF interaction. Frame (e) provides a reference for the location of the new singular points in the region of the compound 3-D interaction of the DF configuration. Frame (f) illustrates the new surface-flow singular points associated with the secondary separation event, and frame (g) illustrates the new surface-flow singular points associated 

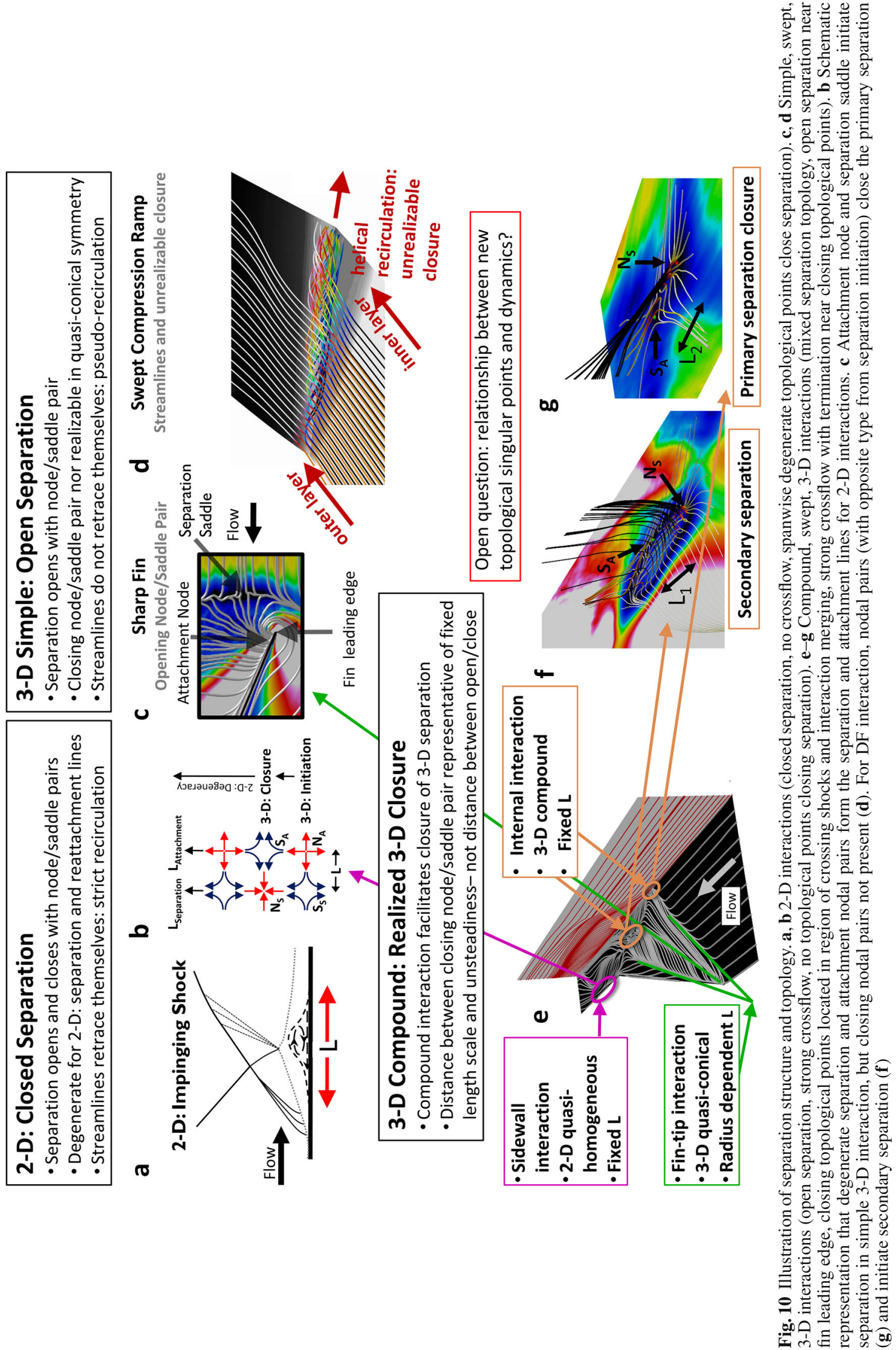
with the closure of the primary separation. The primary separation in the DF interaction is initialized in the same way as the SF interaction (frame c), with an attachment-node/separation-saddle pair near the fin leading edges. The primary separation is then closed by a surface-flow singular-point pair of the opposite type (a separation-node/attachment-saddle pair), as illustrated in frame (g) - thereby providing topological closure to the separation that was absent in the simple swept interactions. Flow that had previously attached near the finplate corner also undergoes a secondary separation event, which is initiated by a separation-node/attachmentsaddle pair, as illustrated in frame (f); like the separation on the symmetry plane, this secondarily separated flow also does not reattach; indeed reattachment of a streamline in 3-D is generally not observed. A host of other singular points occur in the isolator section of the DF interaction, resulting in a very complex structure for the quasi-2-D sidewall interaction as well as the isolator corner flow, both of which merit further investigation. The major conclusion is that key differences in unsteadiness may be anticipated from the fact that the primary separation of the double-fin interaction is topologically closed in the region of the compound 3-D interaction, unlike the infinite simple 3-D interactions.

A third argument may be related to the stability of the flow. The dynamic linear response analyses of spanwise-homogeneous 2-D [3] and simple-swept-3-D interactions [7] have recently demonstrated that the dominant instability of these two interaction classes is of different character. The 2-D interaction exhibits an absolute instability, whereas the simple 3-D interaction exhibits only convective instability. The exhibition of an absolute instability in the spanwise-homogeneous 2-D interaction correlates with the exhibition of lowfrequency bias, and the degenerate closure of the primary separation. All three of these properties are absent in the simple swept interaction suggesting there may be a significant connection among these properties. While the dynamic linear response of the compound 3-D interaction of the DF inlet/isolator configuration has not yet been analyzed, the correlation between topological separation closure and prominent low-frequency spectral biases holds for this interaction as well.

The connection between the structural properties of the separated flow and the dynamics of the DF interaction are finally summarized in the context of Fig. 11. Frames (a, b) describe mean-flow streamlines that are relevant to several separation and attachment events. Seeded farthest from the plate surface, the pseudo-inviscid layer flows through the interaction without significant plate-normal deflection, responding significantly only to compression and expansion through the main shock structure. Closer to the plate surface, the aft-attachment layer attaches to the plate along the primary attachment line and moves downstream after attachment. Closer to the plate surface, the crossflow attachment layer (b) attaches to the plate along the primary attachment line and moves in the crossflow direction; moving toward the interaction centerline, this layer encounters the secondary separation (d) and primary separation closure (e) regions, at which locations this layer separates again from the plate, and does not subsequently reattach. Closest to the plate surface, the inner layer (a) separates from the plate along the primary separation line, and does not subsequently reattach. Frame (c) relates the meanflow streamlines $(a, b)$ and surface-flow singular points $(d, e)$ to the entire surface flow. This illustrates the relationship between the secondary separation (d) and primary separation closure (e) regions to the crossflow attachment layer (b), which separates in these regions.

The wall-pressure spectra along the sidewall/plate-corner (f), quarter-span (g), and half-span (h) lines of the plate are connected to the surface-flow singular points (d, e) and surface flow (i). Frame (i) describes a representative instance of the near-wall streamwise velocity in the low-frequency band of Sect. 4.2 (the low-frequency fluctuations are added to the mean flow resulting in an instance of the low-pass-filtered flow). Here the regions of low-frequency bias in the spectra are connected to corresponding locations in the surface flow. Regions of small streamwise velocity indicating flow separation near the primary separation closure (h), secondary separation $(\mathrm{g}$ ) and quasi-2-D separation in the isolator (f) correspond to the spatial locations of low-frequency biases in the spectra. Therefore, the dynamics of the double-fin inlet/isolator interaction depend on the local separation structure; specifically, regions of the flow exhibiting singular points in the surface flow appear to be correlated with regions of the spectra with low-frequency biases.

\section{Summary and concluding remarks}

The dynamical properties of shock/turbulent-boundary-layer interactions (STBLIs) are connected to the mean flow structure for a sequence of increasingly complex configurations, via the analysis of databases of highfidelity, wall-resolved, large-eddy simulations. The configurations of interest include two-dimensional (2-D) (spanwise-homogeneous), simple, swept, three-dimensional (3-D) (the swept-compression-ramp and sharpfin interactions), and compound 3-D (symmetric double-fin) interactions. The different spectra demonstrate 

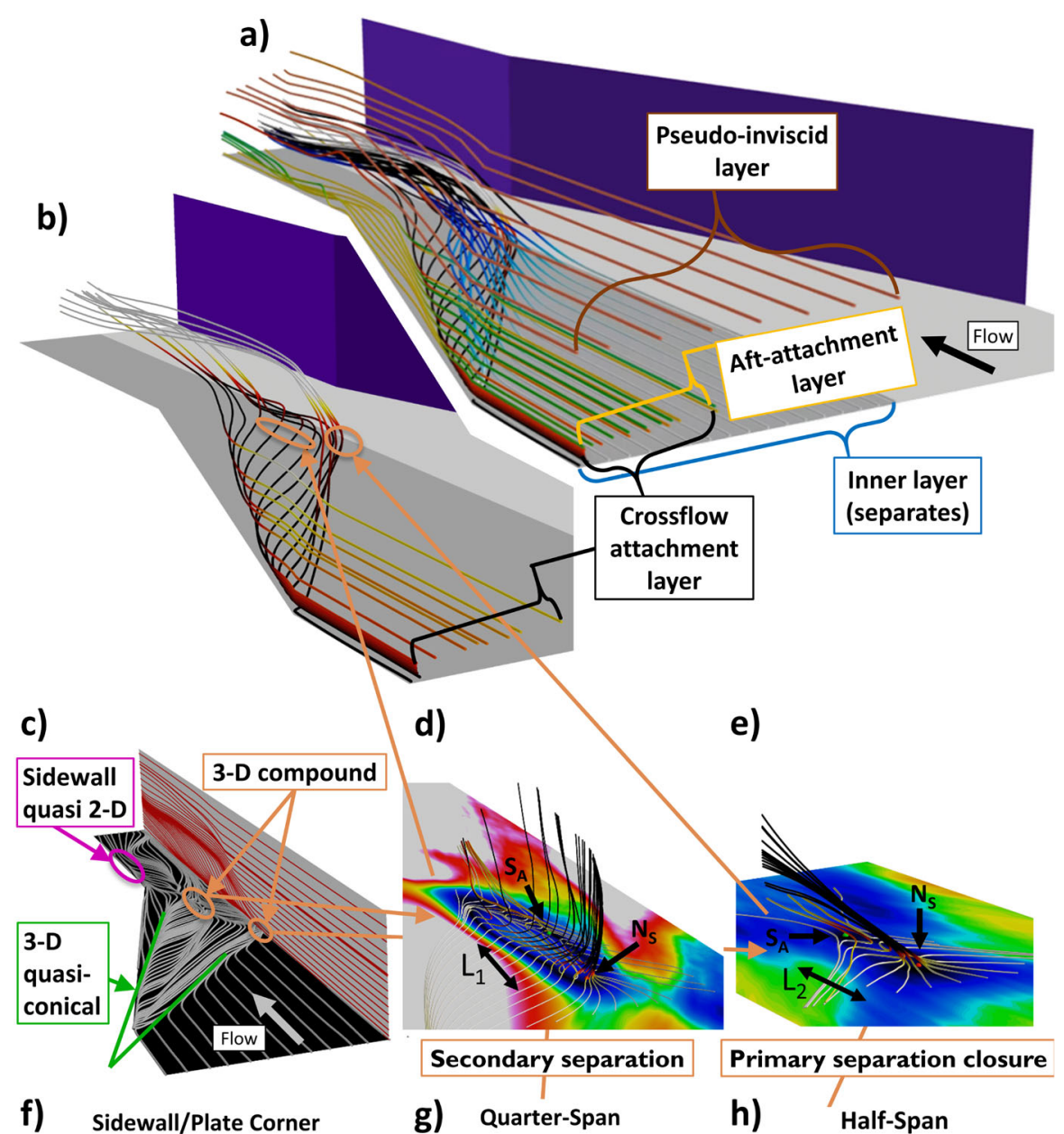

g) Quarter-Span

h)

Half-Span
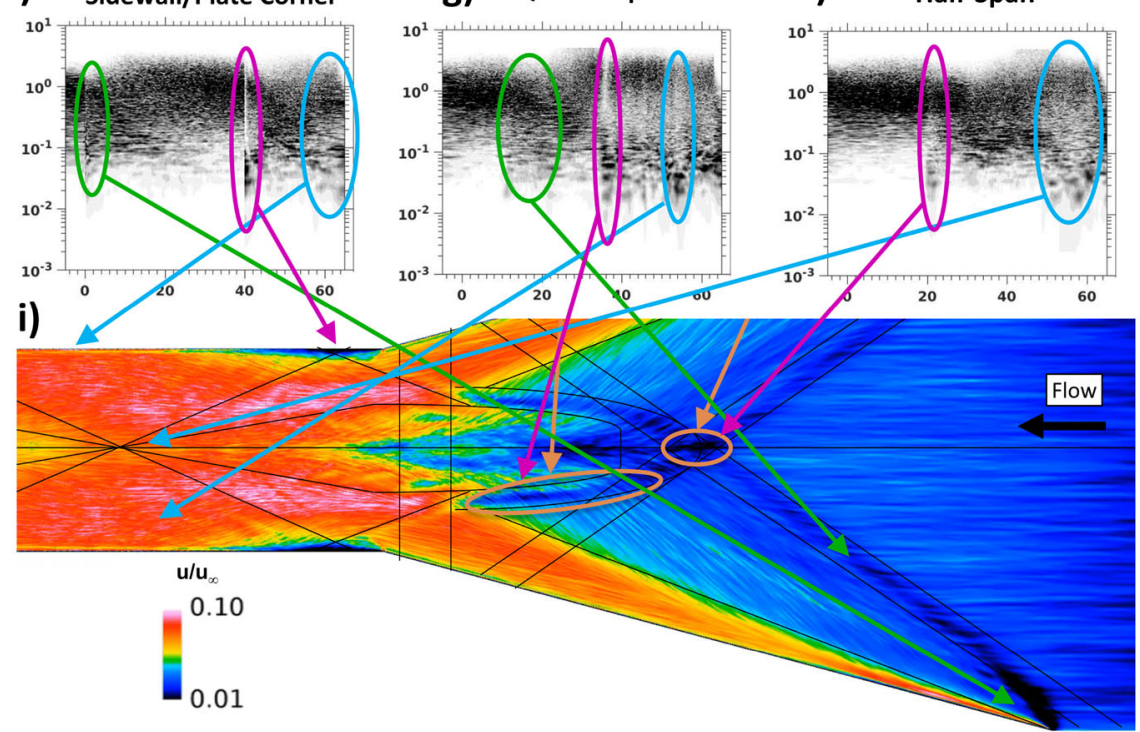

Fig. 11 Relationship between flow structure and unsteadiness in DF interaction. a, b Mean-flow streamlines colored by distance from plate. a Four prominent layers. b Crossflow attachment layer subject to secondary separation. c-e Surface flow visualization (c) connecting the secondary separation (d) and primary separation closure (e) features to crossflow attachment layer of $(\mathbf{b})$. $\mathbf{f}-\mathbf{i}$ Wall-pressure PSD versus streamwise distance along the sidewall/plate-corner (f), quarter-span (g), and half-span (h) lines of the interaction. Annotations connect bands in spectra to flow features identifiable in the near-plate streamwise velocity snapshot (an instance of the low-frequency band: $S t_{\delta} \lesssim 0.02$ ) (i) 
that the dynamical properties of the flow depend strongly on the underlying flow structure. The observed differences in wall-pressure spectra are connected to the structure of the separated flow, including separation length scales, shear layer scaling laws and interaction symmetry. The resulting principles enable the dynamics of more complex interactions to be better intuited from the mean flow structure. Particular focus is applied to the double-fin inlet/isolator configuration, which exhibits aspects of quasi-2-D, simple 3-D, and compound 3-D interactions at various locations, in which the spatial dependence of the spectra may be connected with important flow features.

The description of interaction dynamics is aided by delineating the unsteady phenomena into four frequency bands, each corresponding to prominent dynamical features: very-high-frequency (fine-scale turbulence: $2 \lesssim S t_{\delta}$ ), high-frequency (integral-scale turbulence: $0.2 \lesssim S t_{\delta} \lesssim 2$ )), mid-frequency (coherent shearlayer fluctuations: $0.02 \lesssim S t_{\delta} \lesssim 0.2$ ), and low-frequency (interaction-"breathing" phenomena: $S t_{\delta} \lesssim 0.02$ ) bands. While $S t_{\delta}$ provides a convenient scale for delineating these phenomena, it should be noted that the mid-frequency and low-frequency bands collapse under different normalizations. For simple, swept, 3-D interactions, the mid-frequency band describing coherent fluctuations in the separated shear layers depends on the local properties of the shear layer; this band exhibits both 2 -D $\left(S t_{\delta} \lesssim 0.2\right)$ and 3-D $\left(0.1 \lesssim S t_{L}\right)$ similarity, in which $L$ represents the local arc-length between the separation and attachment lines. The low-frequency band for these 3-D interactions is significantly muted relative to their 2-D counterparts. For spanwise-homogeneous 2-D interactions, the low-frequency band describing the large-scale "breathing" of the interaction collapses based on Strouhal number $\left(S t_{L} \sim 0.03\right.$ ) formed from the mean separation length $(L)$. The mid-frequency band of 2-D interactions necessarily exhibits 2-D similarity.

The relationship between shear-layer scaling laws and mid-frequency unsteadiness in simple, swept, 3-D interactions extends to the early stages of the double-fin interaction, where it resembles two opposing, effectively separate, sharp-fin interactions. The outer separated shear layer exhibits spanwise-homogeneous (2-D) symmetry, consistent with classical free-interaction theory; the coherent fluctuations here are essentially independent of the local separation length and scale with $S t_{\delta}$. The inner shear layer, underneath, contains strong crossflow exhibiting quasi-conical (3-D) symmetry, consistent with conical free-interaction theory; the coherent fluctuations here are dependent on the local separation arc-length, scaling with $S t_{L}$; a similar 3-D scaling that describes the local thickness of the inner shear layer may also be used. Therefore, the lower bound of the mid-frequency unsteadiness associated with the shear layers decreases linearly in frequency with increasing distance from the interaction origin (VCO).

The interaction between the merging sharp-fin flows becomes significant downstream: this region is designated as a compound (as opposed to simple) 3-D interaction. Here, the quasi-conical symmetry of simple swept interactions changes to the centerline symmetry of the compound interaction, with the generation of additional singular points in the surface streamline pattern. These points correspond to flow features that topologically close the primary separation (a phenomenon that cannot occur in an infinite, simple, swept interaction) and initiate secondary separations. These new singular points occur in pairs, and the distance between pairs represents a new characteristic length scale in the flow that may be relevant to the establishment of new frequency scales. The complexity thus evolves from repeating (degenerate) pairs across the span for 2-D interactions, to a single pair in simple 3-D interactions, to multiple pairs in the compound 3-D interaction, in which the quasiconical symmetry is broken, the primary separation exhibits topological closure, and the secondary separation is initiated.

The isolator sidewalls of the double-fin display quasi-2-D interactions, with many similarities to impingingshock interactions with sidewall confinement effects. The singular points corresponding to the opening and closing of these quasi-2-D interactions are not, however, degenerate as with spanwise-homogeneous 2-D interactions, and they can be identified on the sidewalls. Another feature of interest is the complex corner flow that develops in the region of these sidewall interactions and continues downstream through the isolator.

These multiple double-fin flow features manifest a more complex spatial map of the spectra. Upstream of the compound interaction, the wall-pressure spectra are similar to those of the single-fin interactions. In the region of the compound interaction, however, significant low-frequency biases in the spectra are identified. These are prominent in regions where the new surface-flow singular points appear, where primary separation closure or secondary separation occurs. The low-frequency band remains relatively prominent at downstream locations associated with the main shock structure, indicating that the shock structure downstream of the shock crossing has adopted the low-frequency character of the surface flow. A low-frequency bias is also observed in the region of the quasi-2-D sidewall interaction, which is a consequence of the low-frequency bias imparted to the main shock structure during the compound interaction, as well as potential additional mechanisms of low-frequency unsteadiness common to 2-D interactions. Further downstream regions of the isolator exhibit 
a very broad spectrum of unsteadiness, with a somewhat bi-modal character, having clear separation between the high- and low-frequency bands. Thus, complex STBLI configurations involving the interaction of multiple shock systems and the coupling of multiple flow separation and attachment events present a rich new set of complications to the dynamical properties of these interactions that depend on the structure of the separated flow.

Acknowledgements The authors appreciate the sponsorship of the Air Force Office of Scientific Research (Monitor: Dr. S. Popkin, contract FA955014-1-0167) and the Air Force Research Laboratory (AFRL) Collaborative Center for Aeronautical Sciences. Michael Adler has received support through the National Defense Science and Engineering Graduate Fellowship (NDSEG) Program as well as OSU and CTR fellowships; he is currently supported by the U.S. Department of Energy Lawrence Livermore National Laboratory (Monitor: Dr. Andrew. W. Cook, contract DE-AC52-07NA27344). The simulations described were performed with grants of computer time from the Department of Defense HPCMP at the AFRL, ARL, ERDC, and NAVO DSRCs. Some figures have been made with complimentary licenses of FieldView obtained from Intelligent Light through the University Partnership Program. The datasets generated during and/or analyzed during the current study are available from the corresponding author on reasonable request.

Funding Funding was provided by Air Force Office of Scientific Research (Grant Number FA9550-14-1-0167), Air Force Research Laboratory (Grant Number FA8650-19-2-2204).

Open Access This article is licensed under a Creative Commons Attribution 4.0 International License, which permits use, sharing, adaptation, distribution and reproduction in any medium or format, as long as you give appropriate credit to the original author(s) and the source, provide a link to the Creative Commons licence, and indicate if changes were made. The images or other third party material in this article are included in the article's Creative Commons licence, unless indicated otherwise in a credit line to the material. If material is not included in the article's Creative Commons licence and your intended use is not permitted by statutory regulation or exceeds the permitted use, you will need to obtain permission directly from the copyright holder. To view a copy of this licence, visit http://creativecommons.org/licenses/by/4.0/.

\section{References}

1. Adler, M.C.: On the advancement of phenomenological and mechanistic descriptions of unsteadiness in shockwave/turbulent-boundary-layer interactions. Ph.D. Dissertation, The Ohio State University (2019)

2. Adler, M.C., Gaitonde, D.V.: Unsteadiness in swept-compression-ramp shock/turbulent-boundary-layer interactions. In: D 55th AIAA Aerospace Sciences Meeting. AIAA Paper 2017-0987 (2017)

3. Adler, M.C., Gaitonde, D.V.: Dynamic linear response of a shock/turbulent-boundary-layer interaction using constrained perturbations. J. Fluid Mech. 840, 291-341 (2018)

4. Adler, M.C., Gaitonde, D.V.: Unsteadiness in shock/turbulent-boundary-layer interactions with open flow separation. In: 2018 AIAA Aerospace Sciences Meeting. AIAA Paper 2018-2075 (2018)

5. Adler, M.C., Gaitonde, D.V.: Flow similarity in strong swept-shock/turbulent-boundary-layer interactions. AIAA J. 57(4), 1579-1593 (2019)

6. Adler, M.C., Gaitonde, D.V.: Structure, scale, and dynamics of a double-fin shock/turbulent-boundary-layer interaction at Mach 4. In: 2019 AIAA Science and Technology Forum and Exposition. AIAA Paper 2019-0096 (2019)

7. Adler, M.C., Gaitonde, D.V.: Dynamics of strong swept-shock/turbulent-boundary-layer interactions. J. Fluid Mech. 896(A29), 1-35 (2020)

8. Adler, M.C., Gonzalez, D.R., Stack, C.M., Gaitonde, D.V.: Synthetic generation of equilibrium boundary layer turbulence from modeled statistics. Comput. Fluids 165, 127-143 (2018)

9. Agostini, L., Larchevêque, L., Dupont, P.: Mechanism of shock unsteadiness in separated shock/boundary-layer interactions. Phys. Fluids 27(12), 126103 (2015)

10. Agostini, L., Larcheveque, L., Dupont, P., Denieve, J.F., Dussauge, J.P.: Zones of influence and shock motion in a shock/boundary layer interaction. AIAA J. 50(6), 1377-1387 (2012)

11. Arora, N., Ali, M.Y., Alvi, F.S.: Flowfield of a 3-D swept shock boundary layer interaction in a Mach 2 flow. In: 46th AIAA Fluid Dynamics Conference. AIAA Paper 2016-3649 (2016)

12. Arora, N., Mears, L., Alvi, F.S.: Unsteady characteristics of a swept-shock/boundary-layer interaction at Mach 2. AIAA J. 57(10), 4548-4559 (2019)

13. Aubard, G., Gloerfelt, X., Robinet, J.C.: Large-eddy simulation of broadband unsteadiness in a shock/boundary-layer interaction. AIAA J. 51(10), 2395-2409 (2013)

14. Baldwin, A., Mears, L.J., Kumar, R., Alvi, F.S.: Effects of Reynolds number on swept shock-wave/boundary-layer interactions. AIAA J. (2021). https://doi.org/10.2514/1.J060293

15. Baldwin, A.K., Arora, N., Kumar, R., Alvi, F.S.: Effect of Reynolds number on 3-D shock wave boundary layer interactions. In: 46th AIAA Fluid Dynamics Conference. AIAA Paper 2016-3339 (2016)

16. Beresh, S.J., Clemens, N.T., Dolling, D.S.: Relationship between upstream turbulent boundary-layer velocity fluctuations and separation shock unsteadiness. AIAA J. 40(12), 2412-2422 (2002)

17. Bermejo-Moreno, I., Campo, L., Larsson, J., Bodart, J., Helmer, D., Eaton, J.K.: Confinement effects in shock wave/turbulent boundary layer interactions through wall-modelled large-eddy simulations. J. Fluid Mech. 758, 5 (2014)

18. Chapman, D., Kuehn, D., Larson, H.: Investigation of separated flows in supersonic and subsonic streams with emphasis on the effect of transition. Technical Report. NACA, NACA-TR-1356 (1957) 
19. Chapman, G.T., Yates, L.A.: Topology of flow separation on three-dimensional bodies. Appl. Mech. Rev. 44(7), 329-345 (1991)

20. Clemens, N.T., Narayanaswamy, V.: Low-frequency unsteadiness of shock wave/turbulent boundary layer interactions. Ann. Rev. Fluid Mech. 46, 469-492 (2014)

21. Doehrmann, A.C., Padmanabhan, S., Threadgill, J.A., Little, J.C.: Effect of sweep on the mean and unsteady structures of impinging shock/boundary layer interactions. In: 2018 AIAA Aerospace Sciences Meeting. AIAA Paper 2018-2074 (2018)

22. Dolling, D.S.: Fifty years of shock wave/boundary layer interaction research: what next? AIAA J. 39(8), 1517-1531 (2001)

23. Donohoe, S.R., Bannink, W.J.: Surface reflective visualizations of shock-wave/vortex interactions above a delta wing. AIAA J. 35(10), 1568-1573 (1997)

24. Dupont, P., Haddad, C., Debiève, J.F.: Space and time organization in a shock-induced separated boundary layer. J. Fluid Mech. 559, 255-277 (2006)

25. Dussauge, J.P., Dupont, P., Debiève, J.F.: Unsteadiness in shock wave boundary layer interactions with separation. Aerosp. Sci. Technol. 10(2), 85-91 (2006)

26. Erengil, M.E., Dolling, D.S.: Effects of sweepback on unsteady separation in Mach 5 compression ramp interactions. AIAA J. 31(2), 302-311 (1993)

27. Gaitonde, D., Shang, J.S.: Structure of a turbulent double-fin interaction at Mach 4. AIAA J. 33(12), 2250-2258 (1995)

28. Gaitonde, D.V.: Progress in shock wave/boundary layer interactions. Prog. Aerosp. Sci. 72, 80-99 (2015)

29. Gaitonde, D.V., Shang, J.S., Garrison, T.J., Zheltovodov, A.A., Maksimov, A.I.: Three-dimensional turbulent interactions caused by asymmetric crossing-shock configurations. AIAA J. 37(12), 1602-1608 (1999)

30. Gaitonde, D.V., Shang, J.S., Visbal, M.R.: Structure of a double-fin turbulent interaction at high speed. AIAA J. 33(2), 193-200 (1995)

31. Gaitonde, D.V., Visbal, M.R.: High-order schemes for Navier-Stokes equations: algorithm and implementation into FDL3DI. Technical Report. Air Vehicles Directorate, Air Force Research Laboratory, AFRL-VA-WP-TR-1998-3060 (1998)

32. Gaitonde, D.V., Visbal, M.R.: Padé-type higher-order boundary filters for the Navier-Stokes equations. AIAA J. 38(11), 2103-2112 (2000)

33. Ganapathisubramani, B., Clemens, N.T., Dolling, D.S.: Low-frequency dynamics of shock-induced separation in a compression ramp interaction. J. Fluid Mech. 636, 397-425 (2009)

34. Garmann, D.J.: Characterization of the vortex formation and evolution about a revolving wing using high-fidelity simulation. Ph.D. Dissertation, University of Cincinnati (2013)

35. Garrison, T., Settles, G.: Interaction strength and model geometry effects on the structure of crossing-shock wave/turbulent boundary-layer interactions. In: 31st Aerospace Sciences Meeting. AIAA Paper 1993-0780 (1993)

36. Garrison, T.J., Settles, G.S., Narayanswami, N., Knight, D.: Structure of crossing-shock-wave/turbulent-boundary-layer interactions. AIAA J. 31(12), 2204-2211 (1993)

37. Green, J.E.: Interactions between shock waves and turbulent boundary layers. Prog. Aerosp. Sci. 11, $235-340$ (1970)

38. Green, J.E.: Reflexion of an oblique shock wave by a turbulent boundary layer. J. Fluid Mech. 40(1), 81-95 (1970)

39. Gross, A., Little, J.C., Fasel, H.F.: Numerical investigation of shock wave turbulent boundary layer interactions. In: 2018 AIAA Aerospace Sciences Meeting. AIAA Paper 2018-1807 (2018)

40. Gruhn, P., Gülhan, A.: Experimental investigation of a hypersonic inlet with and without sidewall compression. J. Propuls. Power 27(3), 718-729 (2011)

41. Helm, C., Martin, M.P., Dupont, P.: Characterization of the shear layer in a Mach 3 shock/turbulent boundary layer interaction. J. Phys. Conf. Ser. 506, 012013 (2014)

42. Jones, C., Bolton, J., Clifford, C., Thurow, B., Arora, N., Alvi, F.: Single-camera three-dimensional velocity measurement of a fin-generated shock-wave/boundary-layer interaction. AIAA J. 58, 1-13 (2020)

43. Kawai, S., Shankar, S.K., Lele, S.K.: Assessment of localized artificial diffusivity scheme for large-eddy simulation of compressible turbulent flows. J. Comput. Phys. 229(5), 1739-1762 (2010)

44. Knight, D.D., Badekast, D., Horstman, C.C., Settles, G.S.: Quasiconical flowfield structure of the three-dimensional single fin interaction. AIAA J. 30(12), 2809-2816 (1992)

45. Knight, D.D., Horstman, C., Bogdonoff, S.: Structure of supersonic turbulent flow past a swept compression corner. AIAA J. 30(4), 890-896 (1992)

46. Knight, D.D., Yan, H., Panaras, A.G., Zheltovodov, A.: Advances in CFD prediction of shock wave turbulent boundary layer interactions. Progr. Aerosp. Sci. 39, 121-184 (2003)

47. Lele, S.K.: Compact finite difference schemes with spectral-like resolution. J. Comput. Phys. 103(1), 16-42 (1992)

48. Mathew, J., Lechner, R., Foysi, H., Sesterhenn, J., Friedrich, R.: An explicit filtering method for large eddy simulation of compressible flows. Phys. Fluids 15(8), 2279-2289 (2003)

49. Mears, L., Arora, N., Alvi, F.S.: Introducing controlled perturbations in a 3-D swept shock boundary layer interaction. In: 2018 AIAA Aerospace Sciences Meeting. AIAA Paper 2018-2076 (2018)

50. Mears, L.J., Baldwin, A., Ali, M.Y., Kumar, R., Alvi, F.S.: Spatially resolved mean and unsteady surface pressure in swept SBLI using PSP. Exp. Fluids 61(4), 1-14 (2020)

51. Miller, D.S., Wood, R.M.: Leeside flows over delta wings at supersonic speeds. J. Aircr. 21(9), 680-686 (1984)

52. Morgan, B., Duraisamy, K., Nguyen, N., Kawai, S., Lele, S.K.: Flow physics and RANS modelling of oblique shock/turbulent boundary layer interaction. J. Fluid Mech. 729, 231-284 (2013)

53. Mullenix, N., Gaitonde, D.V.: A bandwidth and order optimized WENO interpolation scheme for compressible turbulent flows. In: 49th AIAA Aerospace Sciences Meeting. AIAA Paper 2011-0366 (2011)

54. Nguyen, T., Behr, M., Reinartz, B., Hohn, O., Gülhan, A.: Effects of sidewall compression and relaminarization in a scramjet inlet. J. Propuls. Power 29(3), 628-638 (2013)

55. Nichols, J.W., Larsson, J., Bernardini, M., Pirozzoli, S.: Stability and modal analysis of shock/boundary layer interactions. Theor. Comput. Fluid Dyn. 31, 33-50 (2017)

56. Panaras, A.G.: Review of the physics of swept-shock/boundary layer interactions. Progr. Aerosp. Sci. 32, 173-244 (1996) 
57. Piponniau, S., Dussauge, J.P., Debiève, J.F., Dupont, P.: A simple model for low-frequency unsteadiness in shock-induced separation. J. Fluid Mech. 629, 87-108 (2009)

58. Pirozzoli, S., Grasso, F.: Direct numerical simulation of impinging shock wave/turbulent boundary layer interaction at $\mathrm{M}=$ 2.25. Phys. Fluids 18(6), 065113 (2006)

59. Pirozzoli, S., Larsson, J., Nichols, J.W., Bernardini, M., Morgan, B.E., Lele, S.K.: Analysis of unsteady effects in shock/boundary layer interactions. Cent. Turbul. Res. Proc. Summer Progr. 2010, 153-164 (2010)

60. Plotkin, K.J.: Shock wave oscillation driven by turbulent boundary layer fluctuations. AIAA J. 13(8), 1036-1040 (1975)

61. Poggie, J., Bisek, N.J., Gosse, R.: Resolution effects in compressible, turbulent boundary layer simulations. Comput. Fluids 120, 57-69 (2015)

62. Poggie, J., Porter, K.M.: Flow structure and unsteadiness in a highly confined shock-wave-boundary-layer interaction. Phys. Rev. Fluids 4(2), 024602 (2019)

63. Porter, K.M., Poggie, J.: Selective upstream influence on the unsteadiness of a separated turbulent compression ramp flow. Phys. Fluids 31(1), 016104 (2019)

64. Priebe, S., Martín, M.P.: Low-frequency unsteadiness in shock wave-turbulent boundary layer interaction. J. Fluid Mech. 699, 1-49 (2012)

65. Priebe, S., Tu, J.H., Rowley, C.W., Martín, M.P.: Low-frequency dynamics in a shock-induced separated flow. J. Fluid Mech. 807, 441-477 (2016)

66. Rabey, P.K., Jammy, S.P., Bruce, P.J.K., Sandham, N.D.: Two-dimensional unsteadiness map of oblique shock wave/boundary layer interaction with sidewalls. J. Fluid Mech. (2019). https://doi.org/10.1017/jfm.2019.404

67. Schmisseur, J.D., Dolling, D.S.: Unsteady separation in sharp fin-induced shock wave/turbulent boundary layer interaction at Mach 5. In: 30th Aerospace Sciences Meeting. AIAA Paper 1992-0748 (1992)

68. Schmisseur, J.D., Dolling, D.S.: Fluctuating wall pressures near separation in highly swept turbulent interactions. AIAA J. 32(6), 1151-1157 (1994)

69. Settles, G.S., Degrez, G., Knight, D.D., Dolling, D.S., Delery, J.M., Vandromme, D., Simeonides, G.: Special course on shock-wave/boundary-layer interactions in supersonic and hypersonic flows. Technical Report. NATO Advisory Group for Aerospace Research and Development, AGARD-R-792 (1993)

70. Settles, G.S., Dolling, D.S.: Swept shock wave/boundary-layer interactions. In: Nielsen, J.N., Hemsch, M.J. (eds.) Progress in Astronautics and Aeronautics: Tactical Missile Aerodynamics, vol. 104, pp. 297-379. AIAA (1986)

71. Settles, G.S., Dolling, D.S.: Swept shock/boundary-layer interactions-tutorial and update. In: 28th Aerospace Sciences Meeting. AIAA Paper 1990-0375 (1990)

72. Settles, G.S., Kimmel, R.L.: Similarity of quasiconical shock wave/turbulent boundary-layer interactions. AIAA J. 24(1), 47-53 (1986)

73. Smits, A.J., Dussauge, J.P.: Turbulent Shear Layers in Supersonic Flow. Springer (2006)

74. Souverein, L.J., Dupont, P., Debiève, J.F., Van Oudheusden, B.W., Scarano, F.: Effect of interaction strength on unsteadiness in shock-wave-induced separations. AIAA J. 48(7), 1480-1493 (2010)

75. Souverein, L.J., Van Oudheusden, B.W., Scarano, F., Dupont, P.: Application of a dual-plane particle image velocimetry (dual-PIV) technique for the unsteadiness characterization of a shock wave turbulent boundary layer interaction. Meas. Sci. Technol. 20(7), 074003 (2009)

76. Thivet, F., Knight, D.D., Zheltovodov, A.A., Maksimov, A.I.: Analysis of observed and computed crossing-shockwave/turbulent-boundary-layer interactions. Aerosp. Sci. Technol. 6(1), 3-17 (2002)

77. Threadgill, J.A.S., Little, J.C.: An inviscid analysis of swept oblique shock reflections. J. Fluid Mech. 890(A22), 1-37 (2020)

78. Touber, E., Sandham, N.D.: Large-eddy simulation of low-frequency unsteadiness in a turbulent shock-induced separation bubble. Theor. Comput. Fluid Dyn. 23, 79-107 (2009)

79. Touber, E., Sandham, N.D.: Low-order stochastic modelling of low-frequency motions in reflected shock-wave/boundarylayer interactions. J. Fluid Mech. 671, 417-465 (2011)

80. Vanstone, L., Clemens, N.T.: Proper orthogonal decomposition analysis of swept-ramp shock-wave/boundary-layer unsteadiness at Mach 2. AIAA J. 57(8), 3395-3409 (2019)

81. Vanstone, L., Musta, M.N., Seckin, S., Clemens, N.T.: Experimental study of the mean structure and quasi-conical scaling of a swept-compression-ramp interaction at Mach 2. J. Fluid Mech. 841, 1-27 (2018)

82. Vanstone, L., Saleem, M., Seckin, S., Clemens, N.T.: Effect of upstream boundary layer on unsteadiness of swept-ramp shock/boundary layer interactions at Mach 2. In: 54th AIAA Aerospace Sciences Meeting. AIAA Paper 2016-0076 (2016)

83. Visbal, M.R., Gaitonde, D.V.: On the use of higher-order finite-difference schemes on curvilinear and deforming meshes. J. Comput. Phys. 181(1), 155-185 (2002)

84. Webb, N., Clifford, C., Samimy, M.: Control of oblique shock wave/boundary layer interactions using plasma actuators. Exp. Fluids 54(6), 1-13 (2013)

85. Xiang, X., Babinsky, H.: Corner effects for oblique shock wave/turbulent boundary layer interactions in rectangular channels. J. Fluid Mech. 862, 1060-1083 (2019)

86. Zheltovodov, A.A., Knight, D.D.: Ideal-gas shock wave-turbulent boundary-layer interactions in supersonic flows and their modeling: three-dimensional interactions. In: Babinsky, H., Harvey, J.K. (eds.) Shock Wave-Boundary-Layer Interactions, pp. 202-258. Cambridge University Press (2011)

87. Zuo, F.-Y., Memmolo, A., Huang, G.-P., Pirozzoli, S.: Direct numerical simulation of conical shock wave-turbulent boundary layer interaction. J. Fluid Mech. 877, 167-195 (2019)

Publisher's Note Springer Nature remains neutral with regard to jurisdictional claims in published maps and institutional affiliations. 